LPTENS-03/26

hep-th/0307152

July 2003

\title{
Anomaly cancellation in M-theory: a critical review
}

\author{
Adel Bilal ${ }^{1}$ and Steffen Metzger ${ }^{1,2}$ \\ ${ }^{1}$ CNRS - Laboratoire de Physique Théorique, École Normale Supérieure \\ 24 rue Lhomond, 75231 Paris Cedex 05, France \\ 2 Sektion Physik, Ludwig-Maximilians-Universität \\ Munich, Germany \\ e-mail: adel.bilal@lpt.ens.fr, metzger@physique.ens.fr
}

\begin{abstract}
We carefully review the basic examples of anomaly cancellation in M-theory: the 5-brane anomalies and the anomalies on $S^{1} / \mathbf{Z}_{2}$. This involves cancellation between quantum anomalies and classical inflow from topological terms. To correctly fix all coefficients and signs, proper attention is paid to issues of orientation, chirality and the Euclidean continuation. Independent of the conventions chosen, the ChernSimons and Green-Schwarz terms must always have the same sign. The reanalysis of the reduction to the heterotic string on $S^{1} / \mathbf{Z}_{2}$ yields a surprise: a previously neglected factor forces us to slightly modify the Chern-Simons term, similar to what is needed for cancelling the normal bundle anomaly of the 5-brane. This modification leads to a local cancellation of the anomaly, while maintaining the periodicity on $S^{1}$.
\end{abstract}




\section{Introduction}

Various examples of anomaly cancellation in M-theory are based on an interplay between quantum anomalies on even-dimensional submanifolds and anomaly inflow from the 11-dimensional bulk through a non-invariance of a topological integral like $S_{\mathrm{CS}} \sim \int C \wedge \mathrm{d} C \wedge \mathrm{d} C$ or $S_{\mathrm{GS}} \sim$ $\int G \wedge X_{7}$. For such a cancellation to take place it is clearly crucial that the Chern-Simons and Green-Schwarz terms have the correct signs (and coefficients ${ }^{1}$ ). Also, to compute the quantum anomaly correctly one needs to know exactly the chirality of the fermion zero-modes as well as the self-duality or anti-self-duality of antisymmetric tensor fields on the even-dimensional submanifolds. While in principle straightforward, tracking the various sign conventions of the different computations existing in the literature is quite an enterprise. One can find almost as many choices of one sign as the other for $S_{\mathrm{CS}}$ and $S_{\mathrm{GS}}$ with rather little correlation between both signs. While both choices are possible (and related via $C \rightarrow-C$ ), the relative sign between $S_{\mathrm{CS}}$ and $S_{\mathrm{GS}}$ must be fixed. This motivated us to reanalyse the anomaly cancellation mechanisms in order to determine various consistent conventions of signs and coefficients. Moreover, we also present the Euclidean continuations of all relevant expressions. This turns out to be quite subtle but it is of some advantage since anomalies are typically given in the Euclidean [2].

In the next section, after explaining our conventions, we write the 11-dimensional supergravity action with several parameters related to different choices of normalisations. We use form language, but also write the results explicitly in components. In addition we carefully rewrite the action in Euclidean signature. The subtlety resides in the continuation of topological terms to the Euclidean and it requires to fix some conventions about the orientation of the manifolds.

In section 3, we discuss the five-brane solution, and its bosonic zero-modes, carefully determining the self-duality/ anti-self-duality of the 3-form, which is most important to get the correct sign of the quantum anomaly. Again, this is continued to Euclidean signature.

Section 4 is a quick summary of the results of ref. [2] on gauge and gravitational anomalies (in Euclidean signature), and how they translate back to Minkowski space. Again, we have to be particularly careful about prefactors and signs since we are not simply interested in cancellation between quantum anomalies due to different types of fields but in the cancellation between quantum anomalies and classical inflows from $S_{\mathrm{CS}}$ and $S_{\mathrm{GS}}$.

The anomalies of the 5-brane are discussed in section 5 and their cancellation finally deter-

\footnotetext{
${ }^{1}$ Of course, the coefficient of $S_{\mathrm{CS}}$ was already determined in [1] from imposing supersymmetry.
} 
mines the consistent choices of coefficients and signs for $S_{\mathrm{CS}}$ and $S_{\mathrm{GS}}$. Note that there are two anomalies, the tangent bundle anomaly [3,4] and the normal bundle anomaly [4, 5]. Cancellation of both anomalies gives two independent checks, in particular both correlating the signs of $S_{\mathrm{CS}}$ and $S_{\mathrm{GS}}$.

As a further check, in section 6 , we review the anomaly cancellation in M-theory on $S^{1} / \mathbf{Z}_{2}$ which yields the strongly coupled heterotic string [6]. After a long series of papers, a rather careful analysis [7], insisting on periodicity in the circle coordinate, displayed the basically correct mechanism in the so-called "upstairs" formalism, but it still contained one numerical error. Taking this missing factor into account, anomaly cancellation forces us to slightly modify the Chern-Simons term, much as in the discussion of the normal bundle anomaly of the 5-brane [5] or of anomalies on singular $G_{2}$-manifolds in [8]. Using this modified Chern-Simons term, for the first time, we achieve anomaly cancellation locally, i.e. on each ten-plane separately, while still respecting periodicity on $S^{1}$. In section 7 we summarise our results.

\section{11-dimensional supergravity and the Green-Schwarz term: Minkowskian versus Euclidean}

\subsection{Conventions in Minkowski signature}

We begin by defining our conventions. In the Minkowskian we always use signature $(-,+, \ldots,+)$ and label the coordinates $x^{\mu}, \mu=0, \ldots D-1$. We always choose a right-handed coordinate system such that

$$
\int \sqrt{|g|} \mathrm{d} x^{0} \wedge \mathrm{d} x^{1} \wedge \ldots \wedge \mathrm{d} x^{D-1}=+\int \sqrt{|g|} \mathrm{d}^{D} x \geq 0 .
$$

(With $x^{0}$ being time and for even $D$, this is a non-trivial statement. In particular, for even $D$, if we relabelled time as $x^{0} \rightarrow x^{D}$ then $x^{1}, \ldots x^{D}$ would be a left-handed coordinate system!) We define the $\epsilon$-tensor as

$$
\epsilon_{01 \ldots(D-1)}=+\sqrt{|g|} \Leftrightarrow \epsilon^{01 \ldots(D-1)}=-\frac{1}{\sqrt{|g|}} .
$$

Then

$$
\mathrm{d} x^{\mu_{1}} \wedge \ldots \wedge \mathrm{d} x^{\mu_{D}}=-\epsilon^{\mu_{1} \ldots \mu_{D}} \sqrt{|g|} \mathrm{d}^{D} x .
$$

A $p$-form $\omega$ and its components are related as

$$
\omega=\frac{1}{p !} \omega_{\mu_{1} \ldots \mu_{p}} \mathrm{~d} x^{\mu_{1}} \wedge \ldots \wedge \mathrm{d} x^{\mu_{p}}
$$


and its dual is

$$
{ }^{*} \omega=\frac{1}{p !(D-p) !} \omega_{\mu_{1} \ldots \mu_{p}} \epsilon_{\mu_{p+1} \ldots \mu_{D}}^{\mu_{1} \ldots \mu_{p}} \mathrm{~d} x^{\mu_{p+1}} \wedge \ldots \wedge \mathrm{d} x^{\mu_{D}}
$$

We have ${ }^{*}\left({ }^{*} \omega\right)=(-)^{p(D-p)+1} \omega$ and

$$
\omega \wedge^{*} \omega=\frac{1}{p !} \omega_{\mu_{1} \ldots \mu_{p}} \omega^{\mu_{1} \ldots \mu_{p}} \sqrt{|g|} \mathrm{d}^{D} x .
$$

Finally we note that the components of the $(p+1)$-form $\xi=\mathrm{d} \omega$ are given by

$$
\xi_{\mu_{1} \ldots \mu_{p+1}}=(p+1) \partial_{\left[\mu_{1}\right.} \omega_{\left.\mu_{2} \ldots \mu_{p+1}\right]}
$$

(where the brackets denote anti-symmetrisation with total weight one) and that the divergence of a $p$-form is expressed as

$$
{ }^{*} \mathrm{~d}^{*} \omega=\frac{(-)^{D(p-1)+1}}{(p-1) !} \nabla^{\nu} \omega_{\nu \mu_{1} \ldots \mu_{p-1}} \mathrm{~d} x^{\mu_{1}} \wedge \ldots \wedge \mathrm{d} x^{\mu_{p-1}}
$$

We define the curvature 2-form $R^{a b}=\frac{1}{2} R_{\nu \sigma}^{a b} \mathrm{~d} x^{\nu} \wedge \mathrm{d} x^{\sigma}$ in terms of the spin-connection $\omega^{a b}$ as $R^{a b}=\mathrm{d} \omega^{a b}+\omega_{c}^{a} \wedge \omega^{c b}$. Here $a, b, c=0, \ldots D-1$ are "flat" indices, related to the "curved" ones by the $D$-bein $e_{\mu}^{a}$. The torsion is $T^{a}=\mathrm{d} e^{a}+\omega_{b}^{a} \wedge e^{b}$. The Riemann tensor $R_{\nu \sigma}^{\mu \rho}$ is related to the curvature 2-form via $R_{\nu \sigma}^{a b}=e_{\mu}^{a} e_{\rho}^{b} R_{\nu \sigma}^{\mu \rho}$, and the Ricci tensor is $\mathcal{R}_{\nu}^{\mu}=R_{\nu \rho}^{\mu \rho}$ while the Ricci scalar $\mathcal{R}$ is given by $\mathcal{R}=\mathcal{R}^{\mu}{ }_{\mu}$. With this sign convention, (space-like) spheres have $\mathcal{R}>0$.

\subsection{The 11-dimensional supergravity action and equations of motion in the Minkowskian}

We start with the bosonic part of the 11-dimensional Cremmer-Julia-Scherk supergravity action [1] in the Minkowskian

$$
S_{\mathrm{M}}^{\mathrm{CJS}}=\frac{1}{2 \kappa^{2}} \int \mathrm{d}^{11} x \sqrt{|g|}\left(\gamma \mathcal{R}-\frac{\alpha}{48} G_{\mu \nu \rho \sigma} G^{\mu \nu \rho \sigma}+\frac{\beta}{(144)^{2}} \epsilon^{\mu_{1} \ldots \mu_{11}} C_{\mu_{1} \mu_{2} \mu_{3}} G_{\mu_{4} \ldots \mu_{7}} G_{\mu_{8} \ldots \mu_{11}}\right)
$$

where $\kappa \equiv \kappa_{11}$ is the 11-dimensional gravitational constant, $G_{\mu \nu \rho \sigma}=4 \partial_{[\mu} C_{\nu \rho \sigma]}$ and $\mathcal{R}$ is the Ricci scalar. With our sign convention for the curvature we have $\gamma=+1$. Also, $\alpha$ must be positive. A consistent choice is

$$
\alpha=1, \quad \beta=1, \quad \gamma=1
$$


Other authors define $\mathcal{R}$ with the opposite sign convention which is accounted for by taking $\gamma=-1$. Also, one can always rescale $C$ by a factor $\xi$, changing $\alpha \rightarrow \xi^{2} \alpha$ and $\beta \rightarrow \xi^{3} \beta$. While $\alpha$ remains positive, such a rescaling can change the sign of $\beta$. An invariant combination is $\beta^{2} / \alpha^{3}$ and hence

$$
\frac{\beta^{2}}{\alpha^{3}}=1
$$

(assuming, as we do, $G_{\mu \nu \rho \sigma}=4 \partial_{[\mu} C_{\nu \rho \sigma]}$ or $G=\mathrm{d} C$. Some authors use $G=6 \mathrm{~d} C$ in which case $\beta^{2} / \alpha^{3}=36$.) To facilitate comparison with the literature we explicitly keep $\alpha, \beta$ and $\gamma$ throughout this paper, but the reader may just assume the choice (2.10) and ignore all factors of $\alpha, \beta, \gamma$.

The action (2.9) is rewritten in form language as

$$
S_{\mathrm{M}}^{\mathrm{CJS}}=\frac{1}{2 \kappa^{2}}\left(\gamma \int \mathrm{d}^{11} x \sqrt{|g|} \mathcal{R}-\frac{\alpha}{2} \int G \wedge^{*} G-\frac{\beta}{6} \int C \wedge G \wedge G\right)
$$

where $G=\mathrm{d} C$. Note that the sign in front of $\beta$ has changed with respect to (2.9) in accordance with the minus sign in $(2.3)$.

The equations of motion obtained either from (2.9) or (2.12) by varying the $C$-field can be equivalently written as

$$
\alpha \nabla_{\mu} G^{\mu \nu \rho \sigma}+\frac{\beta}{2 \cdot 4 ! \cdot 4 !} \epsilon^{\nu \rho \sigma \mu_{1} \ldots \mu_{8}} G_{\mu_{1} \ldots \mu_{4}} G_{\mu_{5} \ldots \mu_{8}}=0
$$

or

$$
\alpha \mathrm{d}^{*} G+\frac{\beta}{2} G \wedge G=0
$$

The Einstein equations are

$$
\mathcal{R}_{\mu \nu}=\frac{\alpha}{12 \gamma}\left(G_{\mu \rho \lambda \sigma} G_{\nu}{ }^{\rho \lambda \sigma}-\frac{1}{12} g_{\mu \nu} G_{\rho \lambda \sigma \kappa} G^{\rho \lambda \sigma \kappa}\right)
$$

and can again be written in various ways.

The Green-Schwarz term [9, 3], which is of higher order in $\kappa$, is written as

$$
S_{\mathrm{GS}}=-\epsilon \frac{T_{2}}{2 \pi} \int C \wedge X_{8}=-\epsilon \frac{T_{2}}{2 \pi} \int G \wedge X_{7}
$$

where we assumed that one can freely integrate by parts (no boundaries or singularities), and where

$$
X_{8}=\mathrm{d} X_{7}=\frac{1}{(2 \pi)^{3} 4 !}\left(\frac{1}{8} \operatorname{tr} R^{4}-\frac{1}{32}\left(\operatorname{tr} R^{2}\right)^{2}\right)
$$


Here $T_{2}$ is shorthand for

$$
T_{2}=\left(\frac{2 \pi^{2}}{\kappa^{2}}\right)^{1 / 3}
$$

and will be interpreted as the membrane tension in section 3. The numerical parameter $\epsilon$ will be fixed in section 5 from anomaly cancellation. The Green-Schwarz term modifies the $C$-equation of motion to

$$
\alpha \mathrm{d}^{*} G+\frac{\beta}{2} G \wedge G+\epsilon\left(\frac{2 \kappa^{4}}{\pi}\right)^{\frac{1}{3}} X_{8}=0 .
$$

In Table 1 we give the values of $\gamma, \alpha, \beta$ and $\epsilon$ as well as the convention relating $G$ and $\mathrm{d} C$ of a few references.

\begin{tabular}{|c|c|c|c|c|c|c|c|c|}
\hline reference & signature & $\gamma$ & $\alpha$ & $\beta$ & $\epsilon$ & $G$ & $\frac{\beta^{2}}{\alpha^{3}}$ & $\frac{\epsilon^{3}}{\beta}$ \\
\hline \hline$[1]$ & $+-\ldots-$ & -1 & $2 \kappa^{2}$ & $-2 \sqrt{2} \kappa^{3}$ & & $\mathrm{~d} C$ & 1 & \\
\hline$[10]$ & $-+\ldots+$ & -1 & $2 \kappa^{2}$ & $\mp 12 \sqrt{2} \kappa^{3}$ & & $6 \mathrm{~d} C$ & 36 & \\
\hline$[11]$ & $-+\ldots+$ & 1 & 1 & 1 & & $\mathrm{~d} C$ & 1 & \\
\hline$[12]$ & $-+\ldots+$ & -1 & 2 & $\pm 12 \sqrt{2}$ & & $6 \mathrm{~d} C$ & 36 & \\
\hline$[3]$ & $-+\ldots+$ & 1 & 1 & 1 & -1 & $\mathrm{~d} C$ & 1 & -1 \\
\hline$[13]$ & $-+\ldots+$ & 1 & 4 & -8 & & $\mathrm{~d} C$ & 1 & \\
\hline$[6](2$ nd ref.) & $-+\ldots+$ & -1 & 2 & $\pm 12 \sqrt{2}$ & & $\mathrm{~d} C$ & 36 & \\
\hline$[5]$ & $(-+\ldots+)$ & & & $4 \pi \kappa^{2}$ & & $\mathrm{~d} C$ & & \\
\hline$[14]$ & $(-+\ldots+)$ & & & $4 \pi \kappa^{2}$ & $-\frac{2 \pi}{T_{2}}$ & $\mathrm{~d} C$ & & -1 \\
\hline$[7]$ & $-+\ldots+$ & -1 & 1 & 1 & 1 & $\mathrm{~d} C$ & 1 & 1 \\
\hline$[15]$ & $-+\ldots+$ & 1 & 1 & 1 & 1 & $\mathrm{~d} C$ & 1 & 1 \\
\hline
\end{tabular}

Table 1: Various conventions used in the literature. A value for $\beta$ indicated with a \pm sign means that we were not able to trace the sign convention adopted for $\epsilon^{01 \ldots 10}$ in this reference. Note that for ref. [1] one has to identify $2 \kappa_{\mathrm{CJS}}^{2}=\kappa^{2}$.

\subsection{Continuation to Euclidian signature}

While the functional integral in the Minkowskian contains $e^{i S_{\mathrm{M}}}$, the Euclidean one contains $e^{-S_{\mathrm{E}}}$. This implies

$$
S_{\mathrm{M}}=i S_{\mathrm{E}} \quad, \quad x^{0}=-i x_{\mathrm{E}}^{0} .
$$

However, for a Euclidean manifold $M_{\mathrm{E}}$ it is natural to index the coordinates from 1 to $D$, not from 0 to $D-1$. One could, of course, simply write $i x^{0}=x_{\mathrm{E}}^{0} \equiv x_{\mathrm{E}}^{D}$. The problem then is for even $D=2 n$ that $\mathrm{d} x_{\mathrm{E}}^{0} \wedge \mathrm{d} x^{1} \wedge \ldots \mathrm{d} x^{2 n-1}=-\mathrm{d} x^{1} \wedge \ldots \mathrm{d} x^{2 n-1} \wedge \mathrm{d} x_{\mathrm{E}}^{2 n}$ and if $\left(x_{E}^{0}, \ldots x^{2 n-1}\right)$ was a 
right-handed coordinate system then $\left(x^{1}, \ldots x_{E}^{2 n}\right)$ is a left-handed one. This problem is solved by shifting the indices of the coordinates as

$$
i x^{0}=x_{\mathrm{E}}^{0}=z^{1}, \quad x^{1}=z^{2}, \quad \ldots, \quad x^{D-1}=z^{D} .
$$

This is equivalent to a specific choice of an orientation on the Euclidean manifold $M_{\mathrm{E}}$. In particular, we impose

$$
\int \sqrt{g} \mathrm{~d} z^{1} \wedge \ldots \wedge \mathrm{d} z^{D}=+\int \sqrt{g} \mathrm{~d}^{D} z \geq 0
$$

Then, of course, for any tensor we similarly shift the indices, e.g. $C_{157}=C_{268}^{\mathrm{E}}$ and $C_{034}=i C_{145}^{\mathrm{E}}$. We have $G_{\mu \nu \rho \sigma} G^{\mu \nu \rho \sigma}=G_{j k l m}^{\mathrm{E}} G_{\mathrm{E}}^{j k l m}$ as usual, and for a $p$-form

$$
\omega=\frac{1}{p !} \omega_{\mu_{1} \ldots \mu_{p}} \mathrm{~d} x^{\mu_{1}} \wedge \ldots \wedge \mathrm{d} x^{\mu_{p}}=\frac{1}{p !} \omega_{j_{1} \ldots j_{p}}^{\mathrm{E}} \mathrm{d} z^{j_{1}} \wedge \ldots \wedge \mathrm{d} z^{j_{p}}=\omega^{\mathrm{E}} .
$$

In particular, we have for $p=D$

$$
\int_{M_{\mathrm{M}}} \omega=\int_{M_{\mathrm{E}}} \omega^{\mathrm{E}}
$$

which will be most important below. Finally, note that the Minkowski relations (2.2) and (2.3) become

$$
\mathrm{d} z^{j_{1}} \wedge \ldots \wedge \mathrm{d} z^{j_{D}}=+\epsilon_{\mathrm{E}}^{j_{1} \ldots j_{D}} \sqrt{g} \mathrm{~d}^{D} z \quad \text { with } \quad \epsilon_{\mathrm{E}}^{1 \ldots D}=\frac{1}{\sqrt{g}} .
$$

The dual of a $p$-form $\omega^{\mathrm{E}}$ is defined as in (2.5) but using $\epsilon_{\mathrm{E}}$. It then follows that ${ }^{*}\left({ }^{*} \omega_{\mathrm{E}}\right)=$ $(-)^{p(D-p)} \omega_{\mathrm{E}}$ (with an additional minus sign with respect to the Minkowski relation) and, as in the Minkowskian, $\omega_{\mathrm{E}} \wedge^{*} \omega_{\mathrm{E}}=\frac{1}{p !} \omega_{j_{1} \ldots j_{p}}^{\mathrm{E}} \omega_{\mathrm{E}}^{j_{1} \ldots j_{p}} \sqrt{g} \mathrm{~d}^{D} z$.

We can now readily give the Euclidean action $S_{\mathrm{E}}^{\mathrm{CJS}}$ corresponding to (2.12) or (2.9) via the relations (2.20) and (2.24):

$$
S_{\mathrm{E}}^{\mathrm{CJS}}=\frac{1}{2 \kappa^{2}}\left(-\gamma \int \mathrm{d}^{11} z \sqrt{g} \mathcal{R}_{\mathrm{E}}+\frac{\alpha}{2} \int G_{\mathrm{E}} \wedge{ }^{*} G_{\mathrm{E}}+i \frac{\beta}{6} \int C_{\mathrm{E}} \wedge G_{\mathrm{E}} \wedge G_{\mathrm{E}}\right)
$$

or

$$
S_{\mathrm{E}}^{\mathrm{CJS}}=\frac{1}{2 \kappa^{2}} \int \mathrm{d}^{11} z \sqrt{g}\left(-\gamma \mathcal{R}_{\mathrm{E}}+\frac{\alpha}{48} G_{j k l m}^{\mathrm{E}} G_{\mathrm{E}}^{j k l m}+i \frac{\beta}{(144)^{2}} \epsilon_{\mathrm{E}}^{j_{1} \ldots j_{11}} C_{j_{1} j_{2} j_{3}}^{\mathrm{E}} G_{j_{4} \ldots j_{7}}^{\mathrm{E}} G_{j_{8} \ldots j_{11}}^{\mathrm{E}}\right)
$$

Note that in the Euclidean the sign in front of $\beta$ is the same in (2.26) and (2.27) in accordance with (2.25). While the kinetic terms in the Euclidean action are real, the topological terms are purely imaginary! Similarly, the Euclidean Green-Schwarz term is

$$
S_{\mathrm{GS}}^{\mathrm{E}}=i \epsilon \frac{T_{2}}{2 \pi} \int C_{\mathrm{E}} \wedge X_{8}^{\mathrm{E}}=i \epsilon \frac{T_{2}}{2 \pi} \int G_{\mathrm{E}} \wedge X_{7}^{\mathrm{E}}
$$


We now give the Euclidean equations of motion as derived from the Euclidean action. Einstein's equations do not "see" the topological terms and are identical in form with (2.15), namely

$$
\mathcal{R}_{i j}^{\mathrm{E}}=\frac{\alpha}{12 \gamma}\left(G_{i k l m}^{\mathrm{E}} G_{j}^{\mathrm{E} k l m}-\frac{1}{12} g_{i j}^{\mathrm{E}} G_{k l m n}^{\mathrm{E}} G_{\mathrm{E}}^{k l m n}\right) .
$$

The $C_{\mathrm{E}}$-equation of motion now is (neglecting the contribution from the Green-Schwarz term)

$$
\alpha \mathrm{d}^{*} G_{\mathrm{E}}+i \frac{\beta}{2} G_{\mathrm{E}} \wedge G_{\mathrm{E}}=0
$$

or

$$
\alpha \nabla_{i} G_{\mathrm{E}}^{i j k l}-i \frac{\beta}{2 \cdot 4 ! \cdot 4 !} \epsilon_{\mathrm{E}}^{j k l m_{1} \ldots m_{8}} G_{m_{1} \ldots m_{4}}^{\mathrm{E}} G_{m_{5} \ldots m_{8}}^{\mathrm{E}}=0 .
$$

We already noticed that the topological terms in the Euclidean action are purely imaginary. Actually it is easy to see that an imaginary part of the Euclidean action can only come from terms involving $\sqrt{|g|} \epsilon^{j_{1} \ldots j_{D}}$, i.e. from topological terms. But this is exactly what is needed to obtain anomaly cancellation from an inflow. It is well-known [16] that only the imaginary part of the Euclidean quantum effective action can be anomalous. On the other hand, only the topological terms of the classical action provide anomaly inflow and cancellation can precisely occur if the Euclidean topological terms are imaginary.

Suppose quite generally that under some gauge or local Lorentz transformation the Minkowski classical action has a variation

$$
\delta S_{\mathrm{M}}^{\mathrm{cl}}=\int_{M_{\mathrm{M}}^{2 n}} D_{\mathrm{M}}^{(2 n)}
$$

where $M_{\mathrm{M}}^{2 n}$ is a lower-dimensional manifold of dimension $2 n$. As we have seen, this is equivalent to a variation of the Euclidean action

$$
\delta S_{\mathrm{E}}^{\mathrm{cl}}=-i \int_{M_{\mathrm{E}}^{2 n}} D_{\mathrm{E}}^{(2 n)}
$$

with

$$
D_{\mathrm{M}}^{(2 n)}=D_{\mathrm{E}}^{(2 n)} \equiv D^{(2 n)}
$$

according to (2.23) and (2.24). As we will recall in section 4, the anomalies of the Euclidean effective action are of the form $\left.\delta \Gamma_{\mathrm{E}}\right|_{\text {anomaly }}=-i \int_{M_{\mathrm{E}}^{2 n}} \hat{I}_{2 n}^{1}$ where $\hat{I}_{2 n}^{1}$ is a $2 n$-form. Thus the total variation of $\Gamma_{\mathrm{E}}$ is

$$
\delta \Gamma_{\mathrm{E}}=-i \int_{M_{\mathrm{E}}^{2 n}}\left(\hat{I}_{2 n}^{1}+D^{(2 n)}\right)
$$


Continuing back to Minkowski signature, we get

$$
\delta \Gamma_{\mathrm{M}}=\int_{M_{\mathrm{M}}^{2 n}}\left(\hat{I}_{2 n}^{1}+D^{(2 n)}\right)
$$

where now $\hat{I}_{2 n}^{1}$ is rewritten in Minkowski coordinates according to (2.23). In any case, the condition for anomaly cancellation is the same in Euclidean and Minkowski signature:

$$
\hat{I}_{2 n}^{1}+D^{(2 n)}=0
$$

However, there is a further subtlety that needs to be settled when discussing the relation between the Minkowski and the Euclidean form of the anomaly: we have just seen that the anomaly density $\hat{I}_{2 n}^{1}$ in the Euclidean equals the corresponding anomaly density $\hat{I}_{2 n}^{1}$ in the Minkowskian. This means that we have to know how the chirality matrix $\gamma$ is continued from the Euclidean to the Minkowskian and vice versa. This will be relevant for the $2 n$ dimensional submanifolds. The continuation of the $\gamma$-matrices is dictated by the continuation of the coordinates we have adopted (cf (2.21)):

$$
i \gamma_{\mathrm{M}}^{0}=\gamma_{\mathrm{E}}^{1}, \quad \gamma_{\mathrm{M}}^{1}=\gamma_{\mathrm{E}}^{2}, \quad \ldots \quad \gamma_{\mathrm{M}}^{2 n-1}=\gamma_{\mathrm{E}}^{2 n}
$$

In accordance with ref. [2] we define the Minkowskian and Euclidean chirality matrices $\gamma_{\mathrm{M}}$ and $\gamma_{\mathrm{E}}$ in $2 n$ dimensions as

$$
\gamma_{\mathrm{M}}=i^{n-1} \gamma_{\mathrm{M}}^{0} \ldots \gamma_{\mathrm{M}}^{2 n-1} \quad, \quad \gamma_{\mathrm{E}}=i^{n} \gamma_{\mathrm{E}}^{1} \ldots \gamma_{\mathrm{E}}^{2 n}
$$

Both $\gamma_{\mathrm{M}}$ and $\gamma_{\mathrm{E}}$ are hermitian. Taking into account (2.38) this leads to

$$
\gamma_{\mathrm{M}}=-\gamma_{\mathrm{E}}
$$

i.e. what we call positive chirality in Minkowski space is called negative chirality in Euclidean space and vice versa. This relative minus sign is somewhat unfortunate, but it is necessary to define self-dual $n$-forms from a pair of positive chirality spinors, both in the Minkowskian (with our convention for the $\epsilon$-tensor) and in the Euclidean (with the conventions of [2]). ${ }^{2}$

Indeed, as is well-known, in $2 n=4 k+2$ dimensions, from a pair of spinors of the same chirality one can always construct the components of an $n$-form $H$ by sandwiching $n$ (different)

\footnotetext{
${ }^{2}$ Since we will take [2] as the standard reference for computing anomalies in the Euclidean, we certainly want to use the same convention for $\gamma_{\mathrm{E}}$. On the other hand, we have somewhat more freedom to choose a sign convention for $\gamma_{\mathrm{M}}$. The definition (2.39) of $\gamma_{\mathrm{M}}$ has the further advantage that in $D=10, \gamma_{\mathrm{M}}=\gamma_{\mathrm{M}}^{0} \ldots \gamma_{\mathrm{M}}^{9}$ which is the usual convention used in string theory [10]. Our $\gamma_{\mathrm{M}}$ also agrees with the definition of [11] in $D=2,6$ and 10 (but differs from it by a sign in $D=4$ and 8).
} 
$\gamma$-matrices between the two spinors. In the Minkowskian we call such an $n$-form $H^{\mathrm{M}}$ self-dual if

$$
H_{\mu_{1} \ldots \mu_{n}}^{\mathrm{M}}=+\frac{1}{n !} \epsilon_{\mu_{1} \ldots \mu_{2 n}} H_{\mathrm{M}}^{\mu_{n+1} \ldots \mu_{2 n}}
$$

(with $\epsilon$ given by $(2.2)$ ) and it is obtained from 2 spinors $\psi_{I}(I=1,2)$ satisfying $\gamma_{\mathrm{M}} \psi_{I}=+\psi_{I}$. In the Euclidean $H^{\mathrm{E}}$ is called self-dual if (cf [2])

$$
H_{j_{1} \ldots j_{n}}^{\mathrm{E}}=+\frac{i}{n !} \epsilon_{j_{1} \ldots j_{2 n}}^{\mathrm{E}} H_{\mathrm{E}}^{j_{n+1} \ldots j_{2 n}}
$$

(with $\epsilon^{\mathrm{E}}$ given by $(2.25)$ ) and it is obtained from 2 spinors $\chi_{I}(I=1,2)$ satisfying $\gamma_{\mathrm{E}} \chi_{I}=+\chi_{I}$. With these conventions a self-dual $n$-form in Minkowski space continues to an anti-self-dual $n$-form in Euclidean space, and vice versa, consistent with the fact that positive chirality in Minkowski space continues to negative chirality in Euclidean space. The situation is summarised in Table 2 where each of the four entries corresponds to any of the 3 others.

\begin{tabular}{|c||c|c|}
\hline & Minkowskian & Euclidean \\
\hline \hline spinors & positive chirality & negative chirality \\
\hline$n$-form & self-dual & anti-self-dual \\
\hline
\end{tabular}

Table 2: Correspondences between the (anti-) self-duality of $n$-forms in $2 n=4 k+2$ dimensions and the chirality of the corresponding pair of spinors are given, as well as their Euclidean, resp. Minkowskian continuations.

In conclusion: the anomaly of a positive chirality spinor (or a self-dual $n$-form) in Minkowski

space is given by $\delta \Gamma_{M}=\int_{M_{M}^{2 n}} \hat{I}_{2 n}^{1}$ (cf. (2.36)) but with $\hat{I}_{2 n}^{1}$ the relevant expression of a negative chirality spinor (or an anti-self-dual $n$-form) in Euclidean space.

\section{The 5-brane and its zero-modes}

To determine the exact coefficient of the anomalies on the 6-dimensional 5-brane world-volume, we need to know the chirality of the fermion zero-modes and the sign in the self-duality condition for the 3-form zero-mode living on the 5-brane, respectively anti-5-brane. To do so, we quickly retrace these computations $[17,13]$.

\subsection{The 5-brane solution}

The 5-brane and anti-5-brane are solutions of 11-dimensional supergravity that preserve half of the 32 supersymmetries. The metric is a warped metric preserving Poincaré invariance on 
the $(5+1)$-dimensional world-volume (for flat 5 -branes) and the 4 -form $G$ has a non-vanishing flux through any 4-sphere surrounding the world-volume. This is why the 5-branes are called "magnetic" sources. It will be enough for us to exhibit the bosonic fields only.

We work in Minkowski space and split the coordinates into longitudinal ones $x^{\alpha}, \alpha=0, \ldots 5$ and transverse ones $x^{m} \equiv y^{m}, m=6, \ldots 10$. Then the metric is ${ }^{3}$

$$
\mathrm{d} s^{2}=\Delta(r)^{-1 / 3} \eta_{\alpha \beta} \mathrm{d} x^{\alpha} \mathrm{d} x^{\beta}+\Delta(r)^{2 / 3} \delta_{m n} \mathrm{~d} y^{\mu} \mathrm{d} y^{\nu}
$$

where

$$
\Delta(r)=1+\frac{r_{0}^{3}}{r^{3}} \quad, \quad r=\left(\delta_{m n} y^{m} y^{n}\right)^{1 / 2} \quad, \quad r_{0} \geq 0
$$

(with $\eta_{\alpha \beta}=\operatorname{diag}(-1,1, \ldots 1)$ ). This gives a Ricci tensor (with our sign conventions as described in section 2.1 and corresponding to $\gamma=+1$ )

$$
\mathcal{R}_{\beta}^{\alpha}=-f_{1}(r) \delta_{\beta}^{\alpha}, \quad \mathcal{R}_{n}^{m}=-f_{2}(r) \delta_{n}^{m}-f_{3}(r) \frac{y^{m} y^{n}}{r^{2}}
$$

with

$$
f_{1}(r)=\frac{3}{2} \Delta(r)^{-8 / 3} \frac{r_{0}^{6}}{r^{8}} \quad, \quad f_{2}(r)=-2 f_{1}(r) \quad, \quad f_{3}(r)=3 f_{1}(r) .
$$

The Einstein equations (2.15) are solved by

$$
G_{m n p q}=f(r) \tilde{\epsilon}_{m n p q s} y^{s}, \quad \text { all other } G_{\mu \nu \rho \sigma}=0
$$

with $\widetilde{\epsilon}_{m n p q s}$ completely antisymmetric and $\widetilde{\epsilon}_{678910}=+1$, provided

$$
f(r)^{2}=\frac{9}{\alpha} \frac{r_{0}^{6}}{r^{10}}
$$

(Note that $\alpha>0$ and we used $\gamma=1$. If $\gamma=-1$ one gets the same equations (3.5) and (3.6).) The other equation of motion (2.13) reduces to $\partial_{m}\left(\sqrt{|g|} G^{m n p q}\right)=0$, which is satisfied independent of the detailed form of the function $f(r)$. Hence there are two solutions:

$$
G_{m n p q}= \pm \frac{3 \operatorname{sgn}(\beta)}{\sqrt{\alpha}} \frac{r_{0}^{3}}{r^{5}} \widetilde{\epsilon}_{m n p q s} y^{s}, \quad \text { all other } G_{\mu \nu \rho \sigma}=0 .
$$

The solution with the upper sign $(+)$ is called a 5 -brane and the one with the lower sign $(-)$ an anti-5-brane. If one simply redefines $C \rightarrow-C$ and hence $G \rightarrow-G$ one also has $\beta \rightarrow-\beta$. With the factor of $\operatorname{sgn}(\beta)$ inserted in $(3.7)$ a 5 -brane remains a 5-brane under any rescaling

\footnotetext{
${ }^{3}$ Of course, one should not confuse the longitudinal indices labelled $\alpha, \beta, \gamma, \ldots$ with the coefficients $\alpha, \beta$ and $\gamma$ appearing in the action.
} 
$C \rightarrow \xi C$, even with negative $\xi$. This is desirable since we will assign certain chiral zero-modes to the 5-brane and this chirality (and the corresponding anomaly) should not be changed by a simple rescaling of $C$. The corresponding 4 -form is

$$
G= \pm \operatorname{sgn}(\beta) \frac{r_{0}^{3}}{8 \sqrt{\alpha}} \widetilde{\epsilon}_{\text {mnpqs }} \frac{y^{s}}{r^{5}} \mathrm{~d} y^{m} \wedge \mathrm{d} y^{n} \wedge \mathrm{d} y^{p} \wedge \mathrm{d} y^{q}
$$

and for any 4-sphere in the transverse space surrounding the world-volume we have the "magnetic charge"

$$
\operatorname{sgn}(\beta) \sqrt{\alpha} \int_{S^{4}} G= \pm 3 r_{0}^{3} \operatorname{vol}\left(S^{4}\right)= \pm 8 \pi^{2} r_{0}^{3} .
$$

Hence, for the 5-brane the flux of $\operatorname{sgn}(\beta) G$ is positive and for the anti-5-brane it is negative.

The parameter $r_{0}$ sets the scale for the (anti-) 5-brane solution. One can compute the energy per 5 -volume of the brane, i.e. the 5 -brane tension $T_{5}$. From (2.9) we see that it must be $\frac{1}{\kappa^{2}}$ times a function of $r_{0}$ and hence, on dimensional grounds, $T_{5} \sim \frac{r_{0}^{3}}{\kappa^{2}}$. Including the exact numerical coefficient [17] gives

$$
T_{5}=\frac{8 \pi^{2} r_{0}^{3}}{2 \kappa^{2}}
$$

Thus, the "magnetic charge" of the (anti-) 5 -brane can be expressed as $\operatorname{sgn}(\beta) \sqrt{\alpha} \int_{S^{4}} G=$ $\pm 2 \kappa^{2} T_{5}$. The Dirac quantisation condition between membranes and 5 -branes then relates the membrane tension $T_{2}$ and the 5 -brane tension $T_{5}$ as [18]

$$
T_{2} T_{5}=\frac{2 \pi}{2 \kappa^{2}}
$$

so that $8 \pi^{2} r_{0}^{3}=\frac{2 \pi}{T_{2}}$ and (3.9) can be rewritten as

$$
\operatorname{sgn}(\beta) \sqrt{\alpha} \int_{S^{4}} G= \pm \frac{2 \pi}{T_{2}} .
$$

This is equivalent to the modified Bianchi identity

$$
\operatorname{sgn}(\beta) \sqrt{\alpha} \mathrm{d} G= \pm \frac{2 \pi}{T_{2}} \delta_{W_{6}}^{(5)} \quad\left(\begin{array}{c}
+ \text { for } 5 \text {-brane } \\
- \text { for anti-5-brane }
\end{array}\right)
$$

where $\delta_{W_{6}}^{(5)}$ is a 5 -form Dirac distribution with support on the world-volume $W_{6}$ such that $\int_{M_{11}} \omega_{(6)} \wedge \delta_{W_{6}}^{(5)}=\int_{W_{6}} \omega_{(6)}$. Finally, there is one more relation between the tensions, namely [18]

$$
T_{2}^{2}=2 \pi T_{5}
$$

which together with (3.11) allows us to express $T_{2}$ and $T_{5}$ in terms of $\kappa$ alone, in particular

$$
T_{2}=\left(\frac{2 \pi^{2}}{\kappa^{2}}\right)^{1 / 3}
$$


as already anticipated in (2.18).

To summarise, the 5-brane and anti-5-brane solutions both have a metric given by (3.1). The 4-form $G$ is given by (3.8) and satisfies the Bianchi identity (3.13) with $T_{2}$ given in terms of $\kappa$ by (3.15). The upper sign always corresponds to 5 -branes and the lower sign to anti-5-branes.

\subsection{Zero-modes of the 5-brane}

We will consider the zero-modes of the bosonic equations of motion in the background of the 5-brane solution. The anti-5-brane background can be treated similarly (flipping signs in appropriate places).

Apart from fluctuations describing the position of the 5-brane, there are zero-modes of the $C$-field. A zero-mode is a square-integrable fluctuation $\delta G=\mathrm{d} \delta C$ around the 5-brane solution $G_{0}$ (given by (3.7) or (3.8) with the upper sign) such that $G=G_{0}+\delta G$ still is a solution of (2.13) or (2.14). Of course, $G$ must also solve the Einstein equations to first order in $\delta G$. This will be the case with the same metric if the r.h.s. of (2.15) has no term linear in $\delta G$.

The linearisation of eq. (2.13) around the 5-brane solution (3.7) is

$$
\nabla_{\mu} \delta G^{\mu \nu \rho \sigma}+\frac{\beta}{4 ! 4 ! \alpha} \frac{3 \operatorname{sgn}(\beta) r_{0}^{3}}{\sqrt{\alpha} r^{5}} \epsilon^{\nu \rho \sigma \mu_{1} \ldots \mu_{4} m n p q} \widetilde{\epsilon}_{m n p q s} y^{s} \delta G_{\mu_{1} \ldots \mu_{4}}=0
$$

Since there are only 5 transverse directions, the second term is non-vanishing only if exactly one of the indices $\nu \rho \sigma \mu_{1} \ldots \mu_{4}$ is transverse. It is not too difficult to see that the only solutions are such that all components of $\delta G$ but $\delta G_{m \alpha \beta \gamma}$ vanish. This also ensures that $\delta G$ cannot contribute linearly to the Einstein equations. We take the ansatz [13]

$$
\delta G_{m \alpha \beta \gamma}=\Delta(r)^{-1-\zeta} r^{-5} y^{m} H_{\alpha \beta \gamma}, \quad \text { with } \partial_{n} H_{\alpha \beta \gamma}=0
$$

and use $\sqrt{|g|}=\Delta(r)^{2 / 3}, g^{m n}=\Delta(r)^{-2 / 3} \delta^{m n}, g^{\alpha \beta}=\Delta(r)^{1 / 3} \eta^{\alpha \beta}$, as well as the convention that indices of $H_{\alpha \beta \gamma}$ are raised with $\eta^{\alpha \beta}$ and those of $\delta G_{m \alpha \beta \gamma}$ with $g^{m n}$ and $g^{\alpha \beta}$. This means that $\delta G^{m \alpha \beta \gamma}=\Delta(r)^{-2 / 3-\zeta} r^{-5} y^{m} H^{\alpha \beta \gamma}$. We further need

$$
\epsilon^{\alpha \beta \gamma t \delta \epsilon \varphi m n p q} \widetilde{\epsilon}_{m n p q s}=-\frac{4 !}{\sqrt{|g|}} \delta_{s}^{t} \widetilde{\epsilon}^{\alpha \beta \gamma \delta \epsilon \varphi},
$$

with $\widetilde{\epsilon}^{\alpha \beta \gamma \delta \epsilon \varphi}$ completely antisymmetric and $\widetilde{\epsilon}^{012345}=-1$, i.e. $\widetilde{\epsilon}$ is exactly the $\epsilon$-tensor (as defined in $(2.2))$ for the $(5+1)$-dimensional world-volume with metric $\eta_{\alpha \beta}$. Then, for $(\nu, \rho, \sigma)=(\alpha, \beta, \gamma)$, 
eq. (3.16) becomes ${ }^{4}$

$$
\partial_{m}\left(\Delta(r)^{-\zeta} r^{-5} y^{m}\right) H^{\alpha \beta \gamma}-\frac{|\beta|}{\alpha^{3 / 2}} \frac{r_{0}^{3}}{2} \widetilde{\epsilon}^{\alpha \beta \gamma \delta \epsilon \varphi} \Delta(r)^{-1-\zeta} r^{-8} H_{\delta \epsilon \varphi}=0
$$

Equation (2.11) and $\alpha>0$ imply $\frac{|\beta|}{\alpha^{3 / 2}}=+1$. Since $\partial_{m}\left(\Delta(r)^{-\zeta} r^{-5} y^{m}\right)=+3 \zeta \Delta(r)^{-\zeta-1} r_{0}^{3} r^{-8}$ we finally get

$$
\zeta H^{\alpha \beta \gamma}=\frac{1}{6} \widetilde{\epsilon}^{\alpha \beta \gamma \delta \epsilon \varphi} H_{\delta \epsilon \varphi} .
$$

Consistency of this equation requires either $\zeta=+1$ in which case $H$ is self-dual (cf $(2.41))$ or $\zeta=-1$ in which case $H$ is anti-self-dual.

As mentioned above, the zero-modes must be square-integrable:

$$
\infty>\int \mathrm{d}^{11} x \sqrt{|g|} \delta G_{m \alpha \beta \gamma} \delta G^{m \alpha \beta \gamma}=\frac{8 \pi^{2}}{3} \int_{0}^{\infty} \mathrm{d} r r^{-4} \Delta(r)^{-1-2 \zeta} \int_{W_{6}} \mathrm{~d}^{6} x H_{\alpha \beta \gamma} H^{\alpha \beta \gamma} .
$$

The $r$-integral converges if and only if $\zeta>0$. Thus square-integrability selects $\zeta=+1$ and, hence, $H=\mathrm{d} B$ is a self-dual 3 -form on the world-volume.

To summarise, in Minkowski signature, on a 5-brane, there is a self-dual 3-form $H$ (which continues to an anti-self-dual Euclidean 3-form $H_{\mathrm{E}}$ ), while on an anti-5-brane the 3-form $H$ is anti-self-dual (and continues to a self-dual Euclidean 3-form $H_{\mathrm{E}}$ ). To complete the 6dimensional supermultiplets, we know that the self-dual 3-form is accompanied by two spinors of positive chirality, and the anti-self-dual 3-form by two spinors of negative chirality.

\subsection{The Euclidean 5-brane and its zero-modes}

Instead of determining the zero-modes of the Minkowskian 5-brane and continuing to the Euclidean in the end, one can work in the Euclidean from the beginning. We will now sketch this Euclidean computation and check that the zero-modes of $G_{\mathrm{E}}$ are indeed given by an anti-selfdual (in the Euclidean sense) 3-form on the Euclidean world-volume.

The Euclidean Einstein equations (2.29) are formally the same as the Minkowski ones. On the other hand, eqs. (2.30) and (2.31) are truly complex. Nevertheless, for the 5-brane solution the imaginary part $\sim G_{\mathrm{E}} \wedge G_{\mathrm{E}}$ vanishes. It follows that the Euclidean 5-brane solution is given, in complete analogy with the Minkowski case, by

$$
\begin{aligned}
\mathrm{d} s_{\mathrm{E}}^{2} & =\Delta(r)^{-1 / 3} \delta_{i j} \mathrm{~d} z^{i} \mathrm{~d} z^{j}+\Delta(r)^{2 / 3} \delta_{m n} \mathrm{~d} \tilde{z}^{m} \mathrm{~d} \tilde{z}^{n} \\
G_{m n p q}^{\mathrm{E}} & =\frac{3 \operatorname{sgn}(\beta)}{\sqrt{\alpha}} \frac{r_{0}^{3}}{r^{5}} \hat{\epsilon}_{m n p q s} \tilde{z}^{s}
\end{aligned}
$$

\footnotetext{
${ }^{4}$ For $(\nu, \rho, \sigma)=(m, \beta, \gamma)$ eq. (3.16) gives $\partial_{\alpha} H^{\alpha \beta \gamma}=0$, so that $H_{\alpha \beta \gamma}=3 \partial_{[\alpha} B_{\beta \gamma]}$, as expected.
} 
where now $f, g, h, i, j, k=1, \ldots 6$ label $^{5}$ the longitudinal coordinates $(z)$ and $m, n, p, q, s=$ $7, \ldots 11$ the transverse ones $(\tilde{z})$. The function $\Delta(r)$ is defined as before in terms of $r=$ $\left(\delta_{m n} \tilde{z}^{m} \tilde{z}^{n}\right)^{1 / 2}$. Also, $\hat{\epsilon}_{m n p q s}$ is completely antisymmetric with $\hat{\epsilon}_{7891011}=+1$. $G^{\mathrm{E}}$ satisfies the same Bianchi identity as in the Minkowskian, namely

$$
\operatorname{sgn}(\beta) \sqrt{\alpha} \mathrm{d} G^{\mathrm{E}}=\frac{2 \pi}{T_{2}} \delta_{W_{6}}^{(5)}
$$

Of course, we obtain the Euclidean anti-5-brane by adding an extra minus sign on the r.h.s. of (3.23) and (3.24).

Now we consider zero-modes around this Euclidean 5-brane background (3.23). Inserting the ansatz $\delta G_{m i j k}^{\mathrm{E}}=\Delta(r)^{-1-\zeta} r^{-5} \tilde{z}^{m} H_{i j k}^{\mathrm{E}}$ (all other components vanish) into the linearised eq. (2.31) we get

$$
\nabla_{m} \delta G_{\mathrm{E}}^{m i j k}-i \frac{3 \beta \operatorname{sgn}(\beta) r_{0}^{3}}{4 ! 4 ! \alpha^{3 / 2} r^{5}} 4 \epsilon_{\mathrm{E}}^{i j k t f g h m n p q} \hat{\epsilon}_{m n p q s} \tilde{z}^{s} \delta G_{t f g h}=0
$$

Now eq. (3.18) is replaced by

$$
\epsilon_{\mathrm{E}}^{i j k t f g h m n p q} \hat{\epsilon}_{m n p q s}=-\frac{4 !}{\sqrt{g}} \delta_{s}^{t} \widetilde{\epsilon}_{\mathrm{E}}^{i j k f g h}
$$

where $\tilde{\epsilon}_{\mathrm{E}}^{i j k f g h}$ is the Euclidean $\epsilon$-tensor on the 6-dimensional Euclidean world-volume with metric $\delta_{i j}$. Then the same steps as before lead to

$$
\zeta H_{\mathrm{E}}^{i j k}=-\frac{i}{6} \widetilde{\epsilon}_{\mathrm{E}}^{i j k f g h} H_{f g h}^{\mathrm{E}}
$$

Consistency again requires $\zeta= \pm 1$, and square-integrability selects $\zeta=+1$. We see that the Euclidean 3-form $H_{\mathrm{E}}$ is anti-self dual according to our definition (2.42), in agreement with the previous result.

\section{Gauge and gravitational anomalies of chiral fields}

This section is a summary of the results of [2] where the anomalies for various chiral fields where related to index theory. Again, the emphasis is on conventions, signs and prefactors, since in the sequel we want to add these anomalies to the inflow from the classical action.

\footnotetext{
${ }^{5}$ At this point we ran out of distinct, consistent choices of letters for indices. We hope the reader will forgive us.
} 


\subsection{Conventions}

This entire section is in Euclidean space of dimension $2 n$, and hence we will mostly drop the subscript E. Only at the very end we will discuss the continuation to Minkowski space.

Of course, we use the same conventions as [2] (except that we label space indices $i, j, \ldots$ instead of $\mu, \nu, \ldots$ which we reserved for Minkowski space). The chirality matrix $\gamma \equiv \gamma_{\mathrm{E}}$ was defined in (2.39) and the self-duality condition for an $n$-form in (2.42). The language of differential forms is used throughout. For gauge theory, the gauge fields, field strength and gauge variation are given by

$$
\begin{aligned}
& A=A_{i} \mathrm{~d} z^{i}, \quad A_{i}=A_{i}^{\alpha} \lambda^{\alpha}, \quad\left(\lambda^{\alpha}\right)^{\dagger}=-\lambda^{\alpha}, \\
& F=\mathrm{d} A+A^{2}, \quad \delta_{v} A=\mathrm{D} v=\mathrm{d} v+[A, v] .
\end{aligned}
$$

Thus $F$ is anti-hermitian and differs by an $i$ from a hermitian field strength used by certain authors. ${ }^{6}$ For gravity, one considers the spin connection $\omega_{b}^{a}$ as an $S O(2 n)$-matrix valued 1-form. Similarly, the parameters $\epsilon_{b}^{a}$ of local Lorentz transformations (with $\epsilon^{a b}=-\epsilon^{b a}$ ) are considered as an $S O(2 n)$-matrix. Then

$$
R=\mathrm{d} \omega+\omega^{2}, \quad \delta_{\epsilon} e^{a}=-\epsilon^{a}{ }_{b} e^{b}, \quad \delta_{\epsilon} \omega=\mathrm{D} \epsilon=\mathrm{d} \epsilon+[\omega, \epsilon] .
$$

For spin- $\frac{1}{2}$ fermions the relevant Dirac operator is $\left(E_{a}^{j}\right.$ is the inverse $2 n$-bein)

$$
\not D=E_{a}^{j} \gamma^{a}\left(\partial_{j}+A_{j}+\frac{1}{4} \omega_{c d, j} \gamma^{c d}\right), \quad \gamma^{c d}=\frac{1}{2}\left[\gamma^{c}, \gamma^{d}\right]
$$

\subsection{Index theorems}

The simplest index is that of a positive chirality spin- $\frac{1}{2}$ field. Define $\mathbb{D}_{\frac{1}{2}}=\not D \frac{1+\gamma}{2}$ and

$$
\operatorname{ind}\left(i \not D_{\frac{1}{2}}\right)=\text { number of zero modes of } i \not D_{\frac{1}{2}}-\text { number of zero modes of }\left(i \not D_{\frac{1}{2}}\right)^{\dagger} \text {. }
$$

Then by the Atiyah-Singer index theorem

$$
\operatorname{ind}\left(i \not D_{\frac{1}{2}}\right)=\int_{M_{2 n}}\left[\hat{A}\left(M_{2 n}\right) \operatorname{ch}(F)\right]_{2 n}
$$

where $\operatorname{ch}(F)=\operatorname{tr} \exp \left(\frac{i}{2 \pi} F\right)$ is the Chern character and $\hat{A}\left(M_{2 n}\right)$ is the Dirac genus of the manifold, given below. The subscript $2 n$ indicates to pick only the part which is a $2 n$-form. Note that if the gauge group is $\prod_{k} G_{k}$, then $\operatorname{ch}(F)$ is replaced by $\prod_{k} \operatorname{ch}\left(F_{k}\right)$.

\footnotetext{
${ }^{6}$ For $U(1)$-gauge theories, the usual definition of the covariant derivative is $\partial_{j}+i q \mathcal{A}_{j}$, with $q$ being the charge, and hence $A \simeq i q \mathcal{A}$ and $F \simeq i q \mathcal{F}$ where $\mathcal{F}=\mathrm{d} \mathcal{A}$.
} 
Another important index is that of a positive chirality spin- $\frac{3}{2}$ field. Such a field is obtained from a positive chirality spin- $\frac{1}{2}$ field with an extra vector index by subtracting the spin- $\frac{1}{2}$ part. An extra vector index leads to an additional factor for the index density

$$
\operatorname{tr} \exp \left(\frac{i}{2 \pi} \frac{1}{2} R_{a b} T^{a b}\right)=\operatorname{tr} \exp \left(\frac{i}{2 \pi} R\right)
$$

since the vector representation is $\left(T^{a b}\right)_{c d}=\delta_{c}^{a} \delta_{d}^{b}-\delta_{d}^{a} \delta_{c}^{b}$. Hence

$$
\operatorname{ind}\left(i D_{\frac{3}{2}}\right)=\int_{M_{2 n}}\left[\hat{A}\left(M_{2 n}\right)\left(\operatorname{tr} \exp \left(\frac{i}{2 \pi} R\right)-1\right) \operatorname{ch}(F)\right]_{2 n} .
$$

The third type of fields which lead to anomalies are self-dual or anti-self-dual $n$-forms $H$ in $2 n=4 k+2$ dimensions. Such antisymmetric tensors fields carry no charge w.r.t. the gauge group. As discussed in section 2.3 a self-dual tensor can be constructed from a pair of positive chirality spinors. Correspondingly, the index is $\hat{A}\left(M_{2 n}\right)$ multiplied by $\operatorname{tr} \exp \left(\frac{i}{2 \pi} \frac{1}{2} R_{a b} T^{a b}\right)$, where $T^{a b}=\frac{1}{2} \gamma^{a b}$ as appropriate for the spin- $\frac{1}{2}$ representation. Note that the trace over the spinor representation gives a factor $2^{n}$ in $2 n$ dimensions. There is also an additional factor $\frac{1}{2}$ from the chirality projector of this second spinor and another factor $\frac{1}{2}$ from a reality constraint ( $H$ is real):

$$
\operatorname{ind}\left(i D_{A}\right)=\frac{1}{4} \int_{M_{2 n}}\left[\hat{A}\left(M_{2 n}\right) \operatorname{tr} \exp \left(\frac{i}{2 \pi} \frac{1}{4} R_{a b} \gamma^{a b}\right)\right]_{2 n}=\frac{1}{4} \int_{M_{2 n}}[L(M)]_{2 n} .
$$

$L(M)$ is called the Hirzebruch polynomial, and the subscript on $D_{A}$ stands for "antisymmetric tensor". (Note that, while $\hat{A}\left(M_{2 n}\right) \operatorname{tr} \exp \left(\frac{i}{2 \pi} \frac{1}{4} R_{a b} \gamma^{a b}\right)$ carries an overall factor $2^{n}, L\left(M_{2 n}\right)$ has a factor $2^{k}$ in front of each $2 k$-form part. It is only for $k=n$ that they coincide.)

Of course, the index of a negative chirality (anti-self-dual) field is minus that of the corresponding positive chirality (self-dual) field. Explicitly one has

$$
\begin{aligned}
\operatorname{ch}(F)= & \operatorname{tr} \exp \left(\frac{i}{2 \pi} F\right)=\operatorname{tr} \mathbf{1}+\frac{i}{2 \pi} \operatorname{tr} F+\ldots+\frac{i^{k}}{k !(2 \pi)^{k}} \operatorname{tr} F^{k}+\ldots \\
\hat{A}\left(M_{2 n}\right)= & 1+\frac{1}{(4 \pi)^{2}} \frac{1}{12} \operatorname{tr} R^{2}+\frac{1}{(4 \pi)^{4}}\left[\frac{1}{360} \operatorname{tr} R^{4}+\frac{1}{288}\left(\operatorname{tr} R^{2}\right)^{2}\right] \\
& +\frac{1}{(4 \pi)^{6}}\left[\frac{1}{5670} \operatorname{tr} R^{6}+\frac{1}{4320} \operatorname{tr} R^{4} \operatorname{tr} R^{2}+\frac{1}{10368}\left(\operatorname{tr} R^{2}\right)^{3}\right]+\ldots \\
\hat{A}\left(M_{2 n}\right)\left(\operatorname{tr} \mathrm{e}^{\frac{i}{2 \pi} R}-1\right)= & (2 n-1)+\frac{1}{(4 \pi)^{2}} \frac{2 n-25}{12} \operatorname{tr} R^{2} \\
& +\frac{1}{(4 \pi)^{4}}\left[\frac{2 n+239}{360} \operatorname{tr} R^{4}+\frac{2 n-49}{288}\left(\operatorname{tr} R^{2}\right)^{2}\right]
\end{aligned}
$$




$$
\begin{aligned}
& +\frac{1}{(4 \pi)^{6}}\left[\frac{2 n-505}{5670} \operatorname{tr} R^{6}+\frac{2 n+215}{4320} \operatorname{tr} R^{4} \operatorname{tr} R^{2}+\frac{2 n-73}{10368}\left(\operatorname{tr} R^{2}\right)^{3}\right]+\ldots \\
L\left(M_{2 n}\right)= & 1-\frac{1}{(2 \pi)^{2}} \frac{1}{6} \operatorname{tr} R^{2}+\frac{1}{(2 \pi)^{4}}\left[-\frac{7}{180} \operatorname{tr} R^{4}+\frac{1}{72}\left(\operatorname{tr} R^{2}\right)^{2}\right] \\
& +\frac{1}{(2 \pi)^{6}}\left[-\frac{31}{2835} \operatorname{tr} R^{6}+\frac{7}{1080} \operatorname{tr} R^{4} \operatorname{tr} R^{2}-\frac{1}{1296}\left(\operatorname{tr} R^{2}\right)^{3}\right]+\ldots
\end{aligned}
$$

\subsection{The relation between anomalies in $D=2 n$ and index theorems in $(2 n+2)$ dimensions}

First we need to define what we mean by the anomaly. For the time being we suppose that the classical action is invariant (no inflow), but that the Euclidean quantum effective action $\Gamma_{E}[A]$ has an anomalous variation under the gauge transformation (4.1) with parameter $v$ of the form

$$
\delta_{v} \Gamma_{E}[A]=\int \operatorname{tr} v \mathcal{G}(A)
$$

Local Lorentz anomalies are treated analogously. Note that

$$
\delta_{v} \Gamma_{E}[A]=\int\left(D_{\mu} v\right)^{\alpha} \frac{\delta \Gamma_{E}[A]}{\delta A_{\mu}^{\alpha}}=-\int v^{\alpha}\left(D_{\mu} J^{\mu}\right)^{\alpha}
$$

or $^{7}$

$$
\delta_{v} \Gamma_{E}[A]=\int \operatorname{tr} D_{\mu} v \frac{\delta \Gamma_{E}[A]}{\delta A_{\mu}}=-\int \operatorname{tr} v D_{\mu} \frac{\delta \Gamma_{E}[A]}{\delta A_{\mu}}
$$

so that $\mathcal{G}(A)$ is identified with $-D_{\mu} \frac{\delta \Gamma_{E}[A]}{\delta A_{\mu}}$ or $\mathcal{G}(A)^{\alpha}$ with $-\left(D_{\mu} J^{\mu}\right)^{\alpha}$. We will refer to $\delta_{v} \Gamma_{E}[A]$ as the anomaly, so our anomaly is the negative integrated divergence of the quantum current.

A most important result of [2] is the precise relation between the anomaly in $2 n$ dimensions and index theorems in $2 n+2$ dimensions, which for the pure gauge anomaly of a positive chirality spin- $\frac{1}{2}$ field is (eq. (3.35) of [2]):

$$
\delta_{v} \Gamma_{E}^{\operatorname{spin} \frac{1}{2}}[A]=+\frac{i^{n}}{(2 \pi)^{n}(n+1) !} \int Q_{2 n}^{1}(v, A, F) .
$$

The standard descent equations $\mathrm{d} Q_{2 n}^{1}=\delta_{v} Q_{2 n+1}$ and $\mathrm{d} Q_{2 n+1}=\operatorname{tr} F^{n+1}$ relate $Q_{2 n}^{1}$ to the invariant polynomial tr $F^{n+1}$. Comparing with (4.9) we see that the pure gauge anomaly is thus given by $\delta_{v} \Gamma_{E}^{\operatorname{spin} \frac{1}{2}}[A]=\int I_{2 n}^{1, \text { gauge }}$ with the descent equations $\mathrm{d} I_{2 n}^{1, \text { gauge }}=\delta_{v} I_{2 n+1}^{\text {gauge }}$ and

\footnotetext{
${ }^{7}$ Note that if $A=A^{\alpha} \lambda^{\alpha}, B=B^{\beta} \lambda^{\beta}$ and $\operatorname{tr} \lambda^{\alpha} \lambda^{\beta}=-\delta^{\alpha \beta}$ (the $\lambda^{\alpha}$ are anti-hermitian) then e.g. $\operatorname{tr} A B=$ $-A^{\alpha} B^{\alpha}$ and $\frac{\delta}{\delta A^{\alpha}} \int \operatorname{tr} A B=-B^{\alpha}$. Hence one must define $\frac{\delta}{\delta A}=-\lambda^{\alpha} \frac{\delta}{\delta A^{\alpha}}$ so that $\frac{\delta}{\delta A} \int \operatorname{tr} A B=B$. Another way to see this minus sign in $\frac{\delta}{\delta A}$ is to note that $A^{\alpha}=-\operatorname{tr} \lambda^{\alpha} A$.
} 
$\mathrm{d} I_{2 n+1}^{\text {gauge }}=I_{2 n+2}^{\text {gauge }}$, where $I_{2 n+2}^{\text {gauge }}=-2 \pi i[\operatorname{ch}(F)]_{2 n+2}$. This is immediately generalised to include all gauge and local Lorentz anomalies due to all three types of chiral fields

$$
\begin{aligned}
\delta \Gamma_{E}[A]= & \int I_{2 n}^{1} \\
\mathrm{~d} I_{2 n}^{1}=\delta I_{2 n+1} & , \quad \mathrm{~d} I_{2 n+1}=I_{2 n+2},
\end{aligned}
$$

where $I_{2 n+2}$ equals $-2 \pi i$ times the relevant index density appearing in the index theorem in $2 n+2$ dimensions (corrected by a factor of $\left(-\frac{1}{2}\right)$ in the case of the antisymmetric tensor field, see below). This shows that the Euclidean anomaly is purely imaginary. It is thus convenient to introduce $\hat{I}$ as $I=-i \hat{I}$ so that

$$
\begin{aligned}
& \delta \Gamma_{E}[A]=-i \int \hat{I}_{2 n}^{1} \\
& \mathrm{~d} \hat{I}_{2 n}^{1}=\delta \hat{I}_{2 n+1} \quad, \quad \mathrm{~d} \hat{I}_{2 n+1}=\hat{I}_{2 n+2} .
\end{aligned}
$$

Explicitly we have (always for positive Euclidean chirality, respectively Euclidean self-dual forms)

$$
\begin{aligned}
\hat{I}_{2 n+2}^{\operatorname{spin} \frac{1}{2}} & =2 \pi\left[\hat{A}\left(M_{2 n}\right) \operatorname{ch}(F)\right]_{2 n+2} \\
\hat{I}_{2 n+2}^{\sin \frac{3}{2}} & =2 \pi\left[\hat{A}\left(M_{2 n}\right)\left(\operatorname{tr} \exp \left(\frac{i}{2 \pi} R\right)-1\right) \operatorname{ch}(F)\right]_{2 n+2} \\
\hat{I}_{2 n+2}^{A} & =2 \pi\left[\left(-\frac{1}{2}\right) \frac{1}{4} L\left(M_{2 n}\right)\right]_{2 n+2} .
\end{aligned}
$$

The last equation contains an extra factor $\left(-\frac{1}{2}\right)$ with respect to the index (4.8). The minus sign takes into account the Bose rather than Fermi statistics, and the $\frac{1}{2}$ corrects the $2^{n+1}$ to $2^{n}$ which is the appropriate dimension of the spinor representation on $M_{2 n}$ while the index is computed in $2 n+2$ dimensions. Note that in the cases of interest, the spin- $\frac{3}{2}$ gravitino is not charged under the gauge group and in (4.22) the factor of $\operatorname{ch}(F)$ simply equals 1.

Equations (4.19)-(4.23) together with (4.9)-(4.12) give explicit expressions for the anomalous variation of the Euclidean effective action. In section 2.3. we carefully studied the continuation of topological terms like $\int \hat{I}_{2 n}^{1}$ between Minkowski and Euclidean signature. It follows from equations (2.32)-(2.36) that the anomalous variation of the Minkowskian effective action is given directly by $\hat{I}_{2 n}^{1}$ :

$$
\delta \Gamma_{M}=\int_{M_{2 n}^{M}} \hat{I}_{2 n}^{1}
$$

However, one has to remember that (with our conventions for $\gamma_{M}$ ) the chiralities in Minkowski space and Euclidean space are opposite. While $\hat{I}_{2 n}^{1}$ corresponds to positive chirality in the Euclidean, it corresponds to negative chirality in Minkowski space. 
To facilitate comparison with references [10] and [14] we note that

$$
I([10])=(2 \pi)^{n} \hat{I}_{2 n+2} \quad, \quad I([14])=-\hat{I}_{2 n+2} .
$$

The flip of sign between $I([14])$ and $\hat{I}_{2 n+2}$ is such that $\int I^{1}([14])$ directly gives the variation of the Minkowskian effective action for positive chirality spinors in the Minkowskian (with our definition of $\gamma_{\mathrm{M}}$ ).

\subsection{Tangent and normal bundle anomalies for the 5-brane zero- modes}

We have seen that on the Euclidean 5-brane world volume there live an anti-self-dual 3-form and two negative chirality spinors. While the 3-form cannot couple to gauge fields, the spinors couple to the "SO(5)-gauge" fields of the normal bundle. This coupling occurs via

$$
D_{i}=\partial_{i}+\frac{1}{4} \omega_{a b, i} \gamma^{a b}+\frac{1}{4} \omega_{p q, i} \gamma^{p q}
$$

inherited from the eleven-dimensional spinor. Here $a, b$ and $i$ run from 1 to 6 , while $p, q=$ $7, \ldots 11$. Thus $\omega_{p q, i}$ behaves as an $S O(5)$-gauge field $A_{i}^{\alpha}$ with generators $\lambda^{\alpha} \sim \frac{1}{2} \gamma^{p q}$. We see that the relevant $S O(5)$ representation is the spin representation [4] and hence $\left(R_{p q}=\right.$ $\left.d \omega_{p q}+\omega_{p r} \omega_{r q} \equiv R_{p q}^{\perp}\right)$

$$
\begin{aligned}
F=F^{\alpha} \lambda^{\alpha} & \leftrightarrow \frac{1}{4} R_{p q}^{\perp} \gamma^{p q} \\
\operatorname{ch}(F) & \leftrightarrow \operatorname{tr} \exp \left(\frac{i}{2 \pi} \frac{1}{4} R_{p q}^{\perp} \gamma^{p q}\right) \equiv \operatorname{ch}(S(N))
\end{aligned}
$$

This trace appeared already in (4.8), except that there $R_{a b}$ was the curvature on the manifold (i.e. on the tangent bundle). One has

$$
\operatorname{ch}(S(N))=4\left[1-\frac{1}{(4 \pi)^{2}} \frac{1}{4} \operatorname{tr} R_{\perp}^{2}+\frac{1}{(4 \pi)^{4}}\left[-\frac{1}{24} \operatorname{tr} R_{\perp}^{4}+\frac{1}{32}\left(\operatorname{tr} R_{\perp}^{2}\right)^{2}\right]+\ldots\right]
$$

The relevant anomaly polynomial includes an extra factor $\frac{1}{2}$ from a chirality projector (as in (4.8)) as well as a minus sign for negative chirality. It is $\left(R=\tilde{R}+R_{\perp}\right)$

$$
\begin{aligned}
{\left[-\frac{1}{2} \hat{A}\left(M_{6}\right) \operatorname{ch}(S(N))\right]_{8}=-\frac{2}{(4 \pi)^{4}} } & {\left[\frac{1}{360} \operatorname{tr} \tilde{R}^{4}+\frac{1}{288}\left(\operatorname{tr} \tilde{R}^{2}\right)^{2}\right.} \\
& \left.-\frac{1}{24} \operatorname{tr} R_{\perp}^{4}+\frac{1}{32}\left(\operatorname{tr} R_{\perp}^{2}\right)^{2}-\frac{1}{48} \operatorname{tr} \tilde{R}^{2} \operatorname{tr} R_{\perp}^{2}\right] .
\end{aligned}
$$


The part not involving $R_{\perp}$ is just $-2\left[\hat{A}\left(M_{6}\right)\right]_{8}$ and can be interpreted as the contribution to the tangent bundle anomaly of the two negative chirality spinors on $M_{6}$. Adding the contribution of the anti-self-dual three-form, which is $\left[-\left(-\frac{1}{8}\right) L\left(M_{6}\right)\right]_{8}$ (evaluated using $\left.\tilde{R}\right)$ we get the anomaly on the Euclidean 5-brane as $\delta \Gamma_{E}=-i \int \hat{I}_{6}^{1,5-b r a n e}$ with

$$
\begin{aligned}
\hat{I}_{8}^{5-\text { brane }} & =2 \pi\left[-\frac{1}{2} \hat{A}\left(M_{6}\right) \operatorname{ch}(S(N))+\frac{1}{8} L\left(M_{6}\right)\right]_{8} \\
& =-X_{8}(\tilde{R})-\hat{I}_{8}^{\text {normal }}
\end{aligned}
$$

where $X_{8}$ is given in $(2.17)$ (now with $R \rightarrow \tilde{R}$ ) and

$$
\hat{I}_{8}^{n o r m a l}=\frac{1}{(2 \pi)^{3} 4 !}\left[-\frac{1}{8} \operatorname{tr} R_{\perp}^{4}+\frac{3}{32}\left(\operatorname{tr} R_{\perp}^{2}\right)^{2}-\frac{1}{16} \operatorname{tr} \tilde{R}^{2} \operatorname{tr} R_{\perp}^{2}\right] .
$$

The part $-X_{8}(\tilde{R})$ is called the tangent bundle anomaly and $-\hat{I}_{8}^{\text {normal }}$ the normal bundle anomaly.

\section{Anomaly cancellation for the 5-brane}

In this section we return to Minkowski space. As we have seen, the 5-brane has chiral zeromodes on its 6-dimensional world-volume with its Minkowski anomaly given by

$$
\delta \Gamma_{M}^{1-l o o p}=\int_{W_{6}} \hat{I}_{6}^{1,5-\text { brane }}
$$

where $\hat{I}_{6}^{1,5-b r a n e}$ is the descent of $\hat{I}_{8}^{5-\text { brane }}$ given in $(4.31): I_{8}^{5-\text { brane }}=-X_{8}(\tilde{R})-\hat{I}_{8}^{\text {normal }}$. The tangent bundle anomaly $-X_{8}(\tilde{R})$ is cancelled through inflow from the Green-Schwarz term $\sim \int \wedge \wedge X_{7}(R)$. The latter, however, gives $X_{8}(R)=X_{8}\left(\tilde{R}+R_{\perp}\right)$, not $X_{8}(\tilde{R})$. The difference, as well as the normal bundle anomaly is cancelled through inflow from the Chern-Simons term as was shown in $[4,5]$. As a result, cancellation of the total 5-brane anomaly fixes both coefficients of the Green-Schwarz and Chern-Simons terms. In particular, it establishes a correlation between the two coefficients. Moreover, as we will see, cancellation can only occur if the sign of the anomaly due to the five-brane zero-modes is exactly as in (5.1), (4.31).

Let us first consider inflow from the Green-Schwarz term (2.16). Using the Bianchi identity (3.13) and $\delta X_{7}=\mathrm{d} X_{6}^{1}$ we get

$$
\delta S_{G S}=-\epsilon \frac{T_{2}}{2 \pi} \int G \wedge \mathrm{d} X_{6}^{1}=\epsilon \frac{T_{2}}{2 \pi} \int \mathrm{d} G \wedge X_{6}^{1}=\frac{\epsilon}{\sqrt{\alpha}} \operatorname{sgn}(\beta) \int \delta_{W_{6}}^{(5)} \wedge X_{6}^{1}=\frac{\epsilon}{\sqrt{\alpha}} \operatorname{sgn}(\beta) \int_{W_{6}} X_{6}^{1},
$$


where, as already noted, $X_{6}^{1}$ is $X_{6}^{1}(R)$. This corresponds via descent to an invariant polynomial

$$
\hat{I}_{8}^{G S}=\frac{\epsilon}{\sqrt{\alpha}} \operatorname{sgn}(\beta) X_{8}(R) .
$$

Next, inflow from the Chern-Simons term is more subtle. We review the computation of [5], again paying particular attention to issues of signs and orientation. The two key points in [5] are (i) the regularisation

$$
\delta_{W_{6}}^{(5)} \rightarrow \mathrm{d} \rho \wedge \frac{e_{4}}{2}
$$

where $\rho(r)$ rises monotonically from -1 at $r=0$ to 0 at some finite distance $\tilde{r}$ from the 5brane, and $e_{4}=\mathrm{d} e_{3}$ is a certain angular form with $\int_{S^{4}} \frac{e_{4}}{2}=1$, and (ii) a modification of the Chern-Simons term close to the 5-brane, where $G \neq \mathrm{d} C$.

The regularised Bianchi identity reads

$$
\operatorname{sgn}(\beta) \sqrt{\alpha} \mathrm{d} G=\frac{2 \pi}{T_{2}} \mathrm{~d} \rho \wedge \frac{e_{4}}{2}
$$

which is solved by (requiring regularity at $r=0$ where $e_{4}$ is singular)

$$
\begin{aligned}
G & =\mathrm{d} C+\frac{\operatorname{sgn}(\beta) \pi}{\sqrt{\alpha} T_{2}}\left(2 \mathrm{~d} \rho \wedge \mathrm{d} B-\mathrm{d} \rho \wedge e_{3}\right) \\
& =\frac{\operatorname{sgn}(\beta) \pi}{\sqrt{\alpha} T_{2}} \rho e_{4}+\mathrm{d}\left(C-\frac{\operatorname{sgn}(\beta) \pi}{\sqrt{\alpha} T_{2}}\left(\rho e_{3}+2 \mathrm{~d} \rho \wedge B\right)\right) \\
& \equiv \frac{\operatorname{sgn}(\beta) \pi}{\sqrt{\alpha} T_{2}} \rho e_{4}+\mathrm{d} \widetilde{C} .
\end{aligned}
$$

Under a local Lorentz transformation, $\delta e_{3}=\mathrm{d} e_{2}^{1}$, and $G$ is invariant if $\delta C=0$ and $\delta B=\frac{1}{2} e_{2}^{1}$. Note that [5] include the $\mathrm{d} \rho \wedge B$-term in $C$ and hence get a non-trivial transformation for $C$. If we let $\widetilde{G}=\mathrm{d} \widetilde{C}$ then the modified Chern-Simons term is

$$
\widetilde{S}_{C S}=-\frac{\beta}{12 \kappa^{2}} \lim _{\epsilon \rightarrow 0} \int_{M_{11} \backslash D_{\epsilon} W_{6}} \widetilde{C} \wedge \widetilde{G} \wedge \widetilde{G}
$$

where $M_{11} \backslash D_{\epsilon} W_{6}$ is $M_{11}$ with a small "tubular" region of radius $\epsilon$ around the 5-brane worldvolume cut out. (Of course, this radius $\epsilon$ should not be confused with the $\epsilon$ which is the coefficient of the Green-Schwarz term.) Its boundary is

$$
\partial\left(M_{11} \backslash D_{\epsilon} W_{6}\right)=-S_{\epsilon} W_{6}
$$

where $S_{\epsilon} W_{6}$ is the 4 -sphere bundle over $W_{6}$. Note the minus sign that appears since the orientation of the boundary is opposite to that of the sphere bundle.

Under a local Lorentz transformation $G$ and hence $\widetilde{G}$ are invariant and

$$
\delta \widetilde{C}=-\frac{\operatorname{sgn}(\beta) \pi}{\sqrt{\alpha} T_{2}} \mathrm{~d}\left(\rho e_{2}^{1}\right) .
$$


Inserting this variation into (5.7), and using $\mathrm{d} \widetilde{G}=0$ one picks up a boundary contribution ${ }^{8}$

$$
\delta \widetilde{S}_{C S}=-\frac{\beta \operatorname{sgn}(\beta) \pi}{12 \kappa^{2} \sqrt{\alpha} T_{2}} \lim _{\epsilon \rightarrow 0} \int_{S_{\epsilon} W_{6}} \rho e_{2}^{1} \wedge \widetilde{G} \wedge \widetilde{G} .
$$

In $\widetilde{G}=\mathrm{d} C-\frac{\operatorname{sgn}(\beta) \pi}{\sqrt{\alpha} T_{2}}\left(\mathrm{~d} \rho \wedge e_{3}+\rho e_{4}-2 \mathrm{~d} \rho \wedge \mathrm{d} B\right)$ the terms $\sim \mathrm{d} \rho$ cannot contribute to an integral over $S_{\epsilon} W_{6}$. Also the contribution of the $\mathrm{d} C$-terms vanishes in the limit $\epsilon \rightarrow 0$. Hence the only contribution comes from $[5,19]$

$$
\int_{S_{\epsilon} W_{6}} e_{2}^{1} \wedge e_{4} \wedge e_{4}=2 \int_{W_{6}} p_{2}\left(N W_{6}\right)^{1}
$$

where $p_{2}\left(N W_{6}\right)^{1}$ is related via descent to the second Pontrjagin class $p_{2}\left(N W_{6}\right)$ of the normal bundle. Using $\rho(0)=-1$ and (3.15) we arrive at

$$
\delta \widetilde{S}_{C S}=\frac{\beta \operatorname{sgn}(\beta)}{6 \kappa^{2}}\left(\frac{\pi}{\sqrt{\alpha} T_{2}}\right)^{3} \int_{W_{6}} p_{2}\left(N W_{6}\right)^{1}=\frac{\beta \operatorname{sgn}(\beta)}{\alpha^{3 / 2}} \frac{\pi}{12} \int_{W_{6}} p_{2}\left(N W_{6}\right)^{1} .
$$

This corresponds to an invariant polynomial

$$
\hat{I}_{8}^{C S}=\frac{\beta \operatorname{sgn}(\beta)}{\alpha^{3 / 2}} \frac{\pi}{12} p_{2}\left(N W_{6}\right) .
$$

Using

$$
\begin{aligned}
\frac{\pi}{12} p_{2}\left(N W_{6}\right) & =\frac{1}{(2 \pi)^{3} 4 !}\left(-\frac{1}{4} \operatorname{tr} R_{\perp}^{4}+\frac{1}{8}\left(\operatorname{tr} R_{\perp}^{2}\right)^{2}\right) \\
X_{8}(R) & =X_{8}(\widetilde{R})+\frac{1}{(2 \pi)^{3} 4 !}\left(\frac{1}{8} \operatorname{tr} R_{\perp}^{4}-\frac{1}{32}\left(\operatorname{tr} R_{\perp}^{2}\right)^{2}-\frac{1}{16} \operatorname{tr} \widetilde{R}^{2} \operatorname{tr} R_{\perp}^{2}\right)
\end{aligned}
$$

we find that the total inflow corresponds to

$$
\begin{aligned}
\hat{I}_{8}^{G S}+\hat{I}_{8}^{C S}= & \operatorname{sgn}(\beta) \frac{\epsilon}{\sqrt{\alpha}} X_{8}(\widetilde{R}) \\
& +\frac{\operatorname{sgn}(\beta)}{(2 \pi)^{3} 4 ! \sqrt{\alpha}}\left[\left(\frac{\epsilon}{8}-\frac{\beta}{4 \alpha}\right) \operatorname{tr} R_{\perp}^{4}+\left(\frac{\beta}{8 \alpha}-\frac{\epsilon}{32}\right)\left(\operatorname{tr} R_{\perp}^{2}\right)^{2}-\frac{\epsilon}{16} \operatorname{tr} \widetilde{R}^{2} \operatorname{tr} R_{\perp}^{2}\right] .
\end{aligned}
$$

Now it is easy to study anomaly cancellation: Invariance of the full quantum effective action requires that the sum of (4.31) and (5.15) vanishes. This gives four equations

$$
\begin{aligned}
\operatorname{sgn}(\beta) \frac{\epsilon}{\sqrt{\alpha}}=1 & , \quad \frac{\operatorname{sgn}(\beta)}{\sqrt{\alpha}}\left(\frac{\epsilon}{8}-\frac{\beta}{4 \alpha}\right)=-\frac{1}{8} \\
\frac{\operatorname{sgn}(\beta)}{\sqrt{\alpha}}\left(\frac{\beta}{8 \alpha}-\frac{\epsilon}{32}\right)=\frac{3}{32} & , \quad-\frac{\operatorname{sgn}(\beta)}{\sqrt{\alpha}} \frac{\epsilon}{16}=-\frac{1}{16} .
\end{aligned}
$$

\footnotetext{
${ }^{8}$ We get three minus signs, one from (5.7), (5.8) and (5.9) each. Apparently the one from (5.8) was overlooked in $[5]$.
} 
Recall that under an arbitrary rescaling $C \rightarrow \xi C$ we have $\sqrt{\alpha} \rightarrow|\xi| \sqrt{\alpha}, \beta \rightarrow \xi^{3} \beta$ and $\epsilon \rightarrow \xi \epsilon$. It is satisfying to note that all four equations (5.16) are invariant under this rescaling.

The first equation of (5.16) ensures the cancellation of the tangent bundle anomaly and it fixes

$$
\epsilon=\operatorname{sgn}(\beta) \sqrt{\alpha}
$$

The coefficients of the Green-Schwarz and Chern-Simons terms must have the same sign! Using the relation (2.11) this is equivalent to $\epsilon^{3} / \beta=+1$.

The three other equations (5.16) ensure cancellation of the normal bundle anomaly, and require

$$
|\beta|=\alpha^{3 / 2}, \quad \epsilon=\operatorname{sgn}(\beta) \sqrt{\alpha}
$$

Of course, $|\beta|=\alpha^{3 / 2}$ was already required by supersymmetry [1], cf. eq. (2.11). Fortunately, the second equation selects the same value of $\epsilon$ as the tangent bundle anomaly, and again $\epsilon^{3} / \beta=+1$.

Clearly, anomaly cancellation cannot fix the overall sign of $\beta$ and $\epsilon$ since they can be changed by redefining $C \rightarrow-C$, but the relative sign is fixed. It is also interesting to note that the four conditions (5.16) for anomaly cancellation have enough structure to provide a check that we correctly computed the sign of the one-loop anomaly: suppose we replaced equation (4.31) by

$$
\hat{I}_{8}^{5-\text { brane }}(\eta)=-\eta\left[X_{8}(\widetilde{R})+\hat{I}_{8}^{\text {normal }}\right], \quad \eta= \pm 1
$$

Then equations (5.16) would get an extra factor $\eta= \pm 1$ on their right-hand sides, and equation (5.18) would be replaced by

$$
|\beta|=\alpha^{3 / 2} \eta, \quad \epsilon=\operatorname{sgn}(\beta) \sqrt{\alpha} \eta
$$

This shows that $\eta=+1$, indeed.

At first sight it might seem surprising that a one-loop anomaly of opposite sign could not be cancelled through inflow from the Chern-Simons or Green-Schwarz terms with their signs flipped. As we have seen, such a sign flip merely corresponds to a redefinition of the fields and obviously cannot yield a different inflow. Technically speaking, the factor $\operatorname{sgn}(\beta)$ inserted in the 5-brane Bianchi identity (3.13) ensured that the Euclidean 5-brane always has an anti-self-dual 3 -form zero-mode, independently of the $\operatorname{sign}$ of $\beta$. Also, the inflow involved $\beta \operatorname{sgn}(\beta)=|\beta|$ and $\epsilon \operatorname{sgn}(\beta)=\epsilon \operatorname{sgn}(\epsilon)=|\epsilon|$, and does not depend on the overall sign of $\beta$ and $\epsilon$ (although it is sensitive to the relative sign, as repeatedly emphasised). Alternatively, we could have defined 
the 5 -brane anomaly by $\sqrt{\alpha} \mathrm{d} G=\frac{2 \pi}{T_{2}} \delta_{W_{6}}^{(5)}$ without the $\operatorname{sgn}(\beta)$ inserted. Then, for negative $\beta$, the 5 -brane zero-modes would have had the opposite chirality, reversing the sign of the anomaly, but also the inflow would involve $\beta<0$ and $\epsilon<0$ (without a factor $\operatorname{sgn}(\beta)$ ), and the final result would have been the same. Of course, this simply corresponds to exchanging the roles of 5-branes and anti-5-branes.

In summary, cancellation of all 5-brane anomalies is very powerful and uniquely fixes the coefficients of the Chern-Simons and Green-Schwarz terms as in (5.18), i.e. up to a possible rescaling of the $C$-field. In particular, cancellation of both the tangent and normal bundle anomaly require that the invariant ratio $\frac{\epsilon^{3}}{\beta}$ is

$$
\frac{\epsilon^{3}}{\beta}=+1
$$

\section{Anomaly cancellation on $S^{1} / \mathbf{Z}_{2}$}

When M-theory is compactified on an interval, chiral fields appear on the 10-dimensional boundary planes. Anomaly cancellation through inflow is only possible if the spectrum is that of the $E_{8} \times E_{8}$ heterotic string [6]. Alternatively one can consider M-theory on the orbifold $S^{1} / \mathbf{Z}_{2}$ which has two 10-dimensional fixed planes. These two formalisms are referred to as "downstairs" (the interval) and "upstairs" ( $S^{1}$ with a $\mathbf{Z}_{2}$-projection).

In this section we will show that exactly the relation (5.21) between $\beta$ and $\epsilon$ ensures anomaly cancellation in this case, as well. Furthermore, we show how the sum of Chern-Simons and Green-Schwarz terms reduces to the anomaly-cancelling term of the heterotic string. All this is a non-trivial check of the coefficients $\beta$ and $\epsilon$. We will quickly review the results of [7] and then show that, after correction of a numerical error in [7] a modification of the Chern-Simons term in the vicinity of the fixed planes is necessary. This is similar to the above discussion of the 5-brane normal bundle anomaly.

We start in the downstairs formalism where $S_{C S}$ and $S_{G S}$ are given by integrals over $M_{10}$ times the interval $I=S^{1} / \mathbf{Z}_{2}$,

$$
S_{C S}=-\frac{\beta}{12 \kappa^{2}} \int_{M_{10} \times I} C \wedge G \wedge G, \quad S_{G S}=-\frac{\epsilon}{\left(4 \pi \kappa^{2}\right)^{1 / 3}} \int_{M_{10} \times I} G \wedge X_{7} .
$$

Here $\kappa$ is the eleven-dimensional $\kappa$ as before. This can be rewritten in the upstairs formalism by replacing $\int_{I} \ldots=\frac{1}{2} \int_{S^{1}} \ldots$ and appropriately identifying the fields so that the integrand is 
$\mathbf{Z}_{2}$-even. Defining

$$
\kappa_{\mathrm{U}}^{2}=2 \kappa^{2} \equiv 2 \kappa_{\mathrm{D}}^{2}
$$

one has

$$
\begin{aligned}
& S_{C S}=-\frac{\beta}{12 \kappa_{\mathrm{U}}^{2}} \int_{M_{10} \times S^{1}} C \wedge G \wedge G, \\
& S_{G S}=-\frac{\epsilon}{2^{2 / 3}\left(4 \pi \kappa_{\mathrm{U}}^{2}\right)^{1 / 3}} \int_{M_{10} \times S^{1}} G \wedge X_{7} .
\end{aligned}
$$

Due to the different dependence on $\kappa$, when written in the upstairs formalism, the GreenSchwarz term has an extra factor of $2^{-2 / 3}$. This will be important later on.

As one can see from the Chern-Simons term, $C_{\bar{\mu} \bar{\nu} \bar{\rho}}$ is $\mathbf{Z}_{2}$-odd and $C_{\bar{\mu} \bar{\nu} 10}$ is $\mathbf{Z}_{2}$-even $(\bar{\mu}, \bar{\nu}, \ldots=$ $0, \ldots 9)$. The projection on $\mathbf{Z}_{2}$-even fields then implies e.g. that

$$
C=\tilde{B} \wedge \mathrm{d} x^{10}
$$

with all other components of $C$ projected out. Also, this $\mathbf{Z}_{2}$-projection only leaves half of the components of the eleven-dimensional gravitino [6]. What remains is a ten-dimensional gravitino of positive chirality (in the Minkowskian), together with one negative chirality spin$\frac{1}{2}$ field. Of course, in the Euclidean, this corresponds to one negative chirality spin- $\frac{3}{2}$ and a positive chirality spin- $\frac{1}{2}$ fermion. The 1-loop anomaly due to the eleven-dimensional gravitino on each 10-plane $M_{10}^{A}, A=1,2$ is thus given by

$$
\hat{I}_{12, A}^{\text {gravitino }}=\frac{1}{2} \cdot \frac{1}{2}\left(-\hat{I}_{12}^{\operatorname{spin} \frac{3}{2}}\left(R_{A}\right)+I_{12}^{\operatorname{spin} \frac{1}{2}}\left(R_{A}\right)\right),
$$

where one factor $\frac{1}{2}$ is due to the Majorana condition and the other factor $\frac{1}{2}$ due to the "splitting" of the anomaly between the two fixed planes [6]. $R_{A}$ denotes the curvature two-form on $M_{10}^{A}$ which simply is the eleven-dimensional curvature $R$ with its components tangent to $S^{1}$ suppressed. As is well known, such a polynomial has a $\operatorname{tr} R^{6}$-piece, and one must add an $E_{8}$ vector multiplet in the adjoint representation $(\operatorname{Tr} \mathbf{1}=248)$ with positive chirality (in the Minkowskian) Majorana spinors on each 10-plane. Then on each plane $M_{10}^{A}$ one has a 1-loop anomaly corresponding to

$$
\begin{aligned}
\hat{I}_{12, A} & =\frac{1}{4}\left(-\hat{I}_{12}^{\operatorname{spin} \frac{3}{2}}\left(R_{A}\right)+I_{12}^{\operatorname{spin} \frac{1}{2}}\left(R_{A}\right)\right)-\frac{1}{2} \hat{I}_{12}^{\sin \frac{1}{2}}\left(R_{A}, F_{A}\right) \\
& =I_{4, A}\left[X_{8}\left(R_{A}\right)+\frac{\pi}{3} I_{4, A}^{2}\right],
\end{aligned}
$$


where we used $\operatorname{Tr} F_{A}^{4}=\frac{1}{100}\left(\operatorname{Tr} F_{A}^{2}\right)^{2}, \operatorname{Tr} F_{A}^{6}=\frac{1}{7200}\left(\operatorname{Tr} F_{A}^{2}\right)^{3}$ and defined ${ }^{9}$

$$
I_{4, A}=\frac{1}{(4 \pi)^{2}}\left(\frac{1}{30} \operatorname{Tr} F_{A}^{2}-\frac{1}{2} \operatorname{tr} R_{A}^{2}\right) \equiv \frac{1}{(4 \pi)^{2}}\left(\operatorname{tr} F_{A}^{2}-\frac{1}{2} \operatorname{tr} R_{A}^{2}\right) .
$$

Note that in the small radius limit with $R_{1}=R_{2}=R$ one has

$$
\begin{aligned}
{\left.\left[\hat{I}_{12,1}+\hat{I}_{12,2}\right]\right|_{R_{1}=R_{2}=R} } & =\left(I_{4,1}+I_{4,2}\right)\left[X_{8}(R)+\frac{\pi}{3}\left(I_{4,1}^{2}+I_{4,2}^{2}-I_{4,1} I_{4,2}\right)\right] \\
& \equiv\left(I_{4,1}+I_{4,2}\right) \widehat{X}_{8}\left(R, F_{1}, F_{2}\right)
\end{aligned}
$$

thanks to the algebraic identity $a^{3}+b^{3}=(a+b)\left(a^{2}+b^{2}-a b\right)$. Here $\widehat{X}_{8}$ is the relevant 8 -form that appears in the anomaly-cancelling term of the heterotic string:

$$
\begin{aligned}
& \widehat{X}_{8}\left(R, F_{1}, F_{2}\right)=\frac{1}{(2 \pi)^{3} 4 !}\left(\frac{1}{8} \operatorname{tr} R^{4}+\frac{1}{32}\left(\operatorname{tr} R^{2}\right)^{2}-\frac{1}{8} \operatorname{tr} R^{2}\left(\operatorname{tr} F_{1}^{2}+\operatorname{tr} F_{2}^{2}\right)\right. \\
&\left.+\frac{1}{4}\left(\operatorname{tr} F_{1}^{2}\right)^{2}+\frac{1}{4}\left(\operatorname{tr} F_{2}^{2}\right)^{2}-\frac{1}{4} \operatorname{tr} F_{1}^{2} \operatorname{tr} F_{2}^{2}\right) .
\end{aligned}
$$

The factorised form (6.6) of the anomaly on each ten-plane is a necessary condition to allow for local cancellation through inflow. Clearly, the $I_{4, A} X_{8}$-term has the right form to be cancelled through inflow from the Green-Schwarz term, provided $G$ satisfies a modified Bianchi identity $\mathrm{d} G \sim \sum_{A=1,2} \delta_{A} \wedge I_{4, A}$, where $\delta_{A}$ is a one-form Dirac distribution such that $\int_{M_{10} \times S^{1}} \omega_{(10)} \wedge \delta_{A}=$ $\int_{M_{10}^{A}} \omega_{(10)}$. This is equivalent to prescribing a boundary value for $G$ on the boundary planes in the down-stairs approach. Such a modified Bianchi identity is indeed necessary to maintain supersymmetry in the coupled 11-dimensional supergravity/10-dimensional super-Yang-Mills system [6]. In principle, this allows us to deduce the coefficient $-\zeta$ on the right-hand side of the Bianchi identity in the upstairs approach. It is given by $-(4 \pi)^{2} \frac{\kappa_{\mathrm{U}}^{2}}{\lambda^{2}}$ where $\lambda$ is the (unknown) Yang-Mills coupling constant.

Hence, we start with a Bianchi identity [6]

$$
\mathrm{d} G=-\zeta \sum_{A=1,2} \delta_{A} \wedge I_{4, A}
$$

The variation of the Green-Schwarz term then is

$$
\delta S_{G S}=-\frac{\epsilon}{2^{2 / 3}\left(4 \pi \kappa_{\mathrm{U}}^{2}\right)^{1 / 3}} \int_{M_{10} \times S^{1}} G \wedge \mathrm{d} X_{6}^{1}=-\frac{\epsilon \zeta}{2^{2 / 3}\left(4 \pi \kappa_{\mathrm{U}}^{2}\right)^{1 / 3}} \sum_{A} \int_{M_{10}^{A}} I_{4, A} \wedge X_{6}^{1} .
$$

Provided

$$
\zeta=\frac{2^{2 / 3}\left(4 \pi \kappa_{\mathrm{U}}^{2}\right)^{1 / 3}}{\epsilon}
$$

\footnotetext{
${ }^{9} I_{4, A}$ is exactly what was called $\tilde{I}_{4, i}$ in $[7]$.
} 
$\delta S_{G S}$ corresponds to an invariant polynomial

$$
\hat{I}_{12}^{G S}=-\sum_{A} I_{4, A} \wedge X_{8}\left(R_{A}\right)
$$

As promised, this cancels the part of the anomaly (6.6) involving $X_{8}$. Thus, anomaly cancellation fixes the value of $\zeta$ to be (6.12), thereby determining the value of the 10-dimensional Yang-Mills coupling $\lambda$ in terms of the 11-dimensional gravitational coupling $\kappa$. Although this latter aspect has drawn some attention, one has to realise that the more interesting relation between $\lambda$ and the 10 -dimensional $\kappa_{10}$ involves the (unknown) radius $r_{0}$ of the circle, similarly to the relation between the type IIA string coupling constant and $\kappa$.

To study anomaly inflow from the Chern-Simons term we have to solve the Bianchi identity for $G$ (as we did for the 5-brane). This involves several subtleties, discussed at length in [7]. One important point was to respect periodicity in the circle coordinate $x^{10} \in\left[-\pi r_{0}, \pi r_{0}\right]$ which led to the introduction of two periodic $\mathbf{Z}_{2}$-odd "step" functions $\epsilon_{A}\left(x^{10}\right)$ such that $\epsilon_{1}\left(x^{10}\right)=$ $\operatorname{sgn}\left(x^{10}\right)-\frac{x^{10}}{\pi r_{0}}$ and $\epsilon_{2}\left(x^{10}\right)=\epsilon_{1}\left(x^{10} \pm \pi r_{0}\right)$. They satisfy

$$
\frac{1}{2} \mathrm{~d} \epsilon_{A}=\delta_{A}-\frac{\mathrm{d} x^{10}}{2 \pi r_{0}}
$$

Regularising $\epsilon_{A}$ (and hence $\delta_{A}$ ) properly gives

$$
\delta_{A} \epsilon_{B} \epsilon_{C} \simeq \frac{1}{3} \delta_{A} \delta_{B A} \delta_{C A}
$$

where $\delta_{B A}$ and $\delta_{C A}$ denote the Kronecker symbol. When solving the Bianchi identity (6.10) one can (locally) trade terms $\frac{1}{2} \epsilon_{A} I_{4, A}$ for terms $-\left(\delta_{A}-\frac{\mathrm{d} x^{10}}{2 \pi r_{0}}\right) \omega_{3, A}$, where

$$
\mathrm{d} \omega_{3, A}=I_{4, A}, \quad \delta \omega_{3, A}=\mathrm{d} \omega_{2, A}^{1}
$$

since their difference is a total derivative $\left(\omega_{3, A}\right.$ is given in terms of the Chern-Simons forms on $M_{10}^{A}$ and has no $\mathrm{d} x^{10}$ component). This introduces an arbitrary real parameter $b$ into the solution:

$$
\begin{aligned}
G & =\mathrm{d} C-b \frac{\zeta}{2} \sum_{A}\left(\epsilon_{A} I_{4, A}+\omega_{3, A} \wedge \frac{\mathrm{d} x^{10}}{\pi r_{0}}\right)+(1-b) \zeta \sum_{A} \delta_{A} \wedge \omega_{3, A} \\
& =\mathrm{d}\left(C-b \frac{\zeta}{2} \sum_{A} \epsilon_{A} \omega_{3, A}\right)+\zeta \sum_{A} \delta_{A} \wedge \omega_{3, A} \\
& \equiv \mathrm{d} \widetilde{C}+\zeta \sum_{A} \delta_{A} \wedge \omega_{3, A} .
\end{aligned}
$$


Since $G$ appears in the kinetic term $\sim \int G \wedge{ }^{*} G$, as well as in the energy-momentum tensor, it must be gauge and local Lorentz invariant, $\delta G=0$. This is achieved if [7]

$$
\begin{aligned}
\delta C & =b \zeta \sum_{A=1,2} \omega_{2, A}^{1} \wedge \frac{\mathrm{d} x^{10}}{2 \pi r_{0}}+(1-b) \zeta \sum_{A} \delta_{A} \wedge \omega_{2, A}^{1} \\
\Leftrightarrow \delta \widetilde{C} & =\mathrm{d}\left(-b \frac{\zeta}{2} \sum_{A} \epsilon_{A} \omega_{2, A}^{1}\right)+\zeta \sum_{A} \delta_{A} \wedge \omega_{2, A}^{1} .
\end{aligned}
$$

In [7] several arguments were given in favour of one particular value of $b$, namely $b=1$, since only then $G$ is globally well-defined. Furthermore, the higher Fourier modes of $C_{\bar{\mu} \bar{\nu} 10}$ are gauge invariant only for this value of $b$, which is a necessary condition for a safe truncation to the perturbative heterotic string. Last, but not least, it is only for $b=1$ that $G$ has no terms involving $\delta_{A}$ which would lead to divergent pieces in the kinetic term $\int G \wedge^{*} G$. Nevertheless, we will keep this parameter $b$ for the time being and show in the end that anomaly cancellation also requires $b=1$.

Note that, although $G \neq \mathrm{d} C$, we still have $G=\mathrm{d} \widetilde{C}$ as long as we stay away from the fixed planes. This motivates us to introduce a modified Chern-Simons term similar to what was done in section 5 for the 5 -brane or in [8] when discussing M-theory on singular $G_{2}$-manifolds. We take

$$
\widetilde{S}_{C S}=-\frac{\beta}{12 \kappa_{\mathrm{U}}^{2}} \int_{M_{10} \times S^{1}} \widetilde{C} \wedge G \wedge G
$$

which away from the fixed planes is just $\sim \int \widetilde{C} \wedge \mathrm{d} \widetilde{C} \wedge \mathrm{d} \widetilde{C}$. Then

$$
\begin{aligned}
\delta \widetilde{S}_{C S}=-\frac{\beta}{12 \kappa_{\mathrm{U}}^{2}} \int_{M_{10} \times S^{1}} \delta \widetilde{C} \wedge G \wedge G \\
=-\frac{\beta}{12 \kappa_{\mathrm{U}}^{2}} \int_{M_{10} \times S^{1}}\left[\mathrm{~d}\left(-b \frac{\zeta}{2} \sum_{A} \epsilon_{A} \omega_{2, A}^{1}\right) \wedge 2 \mathrm{~d} \widetilde{C} \wedge \zeta \sum_{C} \delta_{C} \wedge \omega_{3, C}\right. \\
\left.+\zeta \sum_{A} \delta_{A} \wedge \omega_{2, A}^{1} \wedge \mathrm{d} \widetilde{C} \wedge \mathrm{d} \widetilde{C}\right]
\end{aligned}
$$

Note that we can freely integrate by parts (we assume that $M_{10}$ has no boundary). Furthermore, since both $\delta_{A}$ and $\mathrm{d} C=\mathrm{d} \tilde{B} \wedge \mathrm{d} x^{10}$ always contain a $\mathrm{d} x^{10}$, on the r.h.s of eq. (6.20) one can replace $\mathrm{d} \widetilde{C} \rightarrow-b \frac{\zeta}{2} \sum_{B} \epsilon_{B} I_{4, B}$, so that

$$
\delta \widetilde{S}_{C S}=-\frac{\beta}{12 \kappa_{\mathrm{U}}^{2}} b^{2}\left(\frac{\zeta^{3}}{4}\right) \int_{M_{10} \times S^{1}} \sum_{A, B, C}\left(2 \epsilon_{A} \epsilon_{B} \delta_{C}+\delta_{A} \epsilon_{B} \epsilon_{C}\right) \omega_{2, A}^{1} \wedge I_{4, B} \wedge I_{4, C} .
$$

The modified Chern-Simons term contributes three terms $\delta \epsilon \epsilon$. This factor of 3 was absent in [7] where inflow from the unmodified Chern-Simons term was computed. Also the result of [7] 
was obtained only after using $\int_{S^{1}} \mathrm{~d} x^{10} \epsilon_{A} \epsilon_{B}=\pi r_{0}\left(\delta_{A B}-\frac{1}{3}\right)$ which somewhat obscured the local character of anomaly cancellation. Now, however, due to the explicit $\delta_{A}$ one-forms, the inflow from $\widetilde{S}_{C S}$ is localised on the 10-planes $M_{10}^{A}$. Using (6.15) we find

$$
\delta \widetilde{S}_{C S}=-\frac{\beta \zeta^{3}}{48 \kappa_{\mathrm{U}}^{2}} b^{2} \sum_{A=1,2} \int_{M_{10}^{A}} \omega_{2, A}^{1} \wedge I_{4, A} \wedge I_{4, A} .
$$

Upon inserting the value of $\zeta$, equation (6.12), we see that this corresponds to an invariant polynomial

$$
\hat{I}_{12}^{\widetilde{C S}}=-\frac{\beta}{\epsilon^{3}} b^{2} \frac{\pi}{3} \sum_{A=1,2} I_{4, A}^{3} .
$$

This cancels the remaining piece of the anomaly (6.6) precisely if

$$
b^{2}=\frac{\epsilon^{3}}{\beta} .
$$

Whatever the (real) value of $b$ is, this shows again that the coefficients of the Green-Schwarz and Chern-Simons terms, $\epsilon$ and $\beta$ must have the same sign. Moreover, we noted already that only $b=1$ is consistent, so that anomaly cancellation in the present case again confirms

$$
\frac{\epsilon^{3}}{\beta}=+1
$$

Conversely, using $\frac{\epsilon^{3}}{\beta}=1$ from anomaly cancellation for the 5-brane, we can conclude that anomaly cancellation on $S^{1} / \mathbf{Z}_{2}$ requires $b=1$, as argued in [7]. ${ }^{10}$

Thus we have shown that all the anomalies are cancelled locally through inflow from the Green-Schwarz $^{11}$ and (modified) Chern-Simons terms with exactly the same coefficients as already selected from cancellation of the 5-brane anomalies.

Finally, it is easy to show that in the small radius limit $\left(r_{0} \rightarrow 0\right)$ the sum $S_{G S}+\widetilde{S}_{C S}$ exactly reproduces the heterotic Green-Schwarz term. In this limit $X_{8}(R)$ and $X_{7}(R)$ are independent of $x^{10}$ and have no $\mathrm{d} x^{10}$ components. From $C=\tilde{B} \wedge \mathrm{d} x^{10}$ and $\delta C$ given in (6.18) we identify the correctly normalised heterotic $B$-field as the zero mode of $\tilde{B}$ times $\frac{(4 \pi)^{2}}{\zeta} 2 \pi r_{0}$ :

$$
B=\frac{(4 \pi)^{2}}{\zeta} \int_{S^{1}} \tilde{B} \wedge \mathrm{d} x^{10}, \quad \delta B=(4 \pi)^{2} \sum_{A} \omega_{2, A}^{1}=\omega_{2, Y M}^{1}-\omega_{2, L}^{1}
$$

\footnotetext{
${ }^{10}$ In [7] inflow from the unmodified Chern-Simons term was computed. This is three times smaller than (6.22). Also the factor $2^{2 / 3}$ in $\zeta$ was missing, so that the overall inflow $\delta S_{C S}$ appeared 12 times smaller. This discrepancy remained unnoticed since the anomaly cancellation condition was expressed as $\frac{(4 \pi)^{5} \kappa^{4} b^{2}}{12 \lambda^{6}}=1$. It is only after relating $\frac{\lambda^{2}}{\kappa^{2}}$ to the coefficient of the Green-Schwarz term that one can use $\frac{(4 \pi)^{5} \kappa^{4}}{\lambda^{6}}=1$ and then $\frac{b^{2}}{12}=1$ clearly is in conflict with $b=1$.

${ }^{11} \mathrm{It}$ is interesting to note that $\widetilde{S}_{G S}=-\frac{\epsilon}{2^{2 / 3}\left(4 \pi \kappa_{\mathrm{U}}^{2}\right)^{1 / 3}} \int_{M_{10} \times S^{1}} \widetilde{C} \wedge X_{8}$ would have led to the same result.
} 
where $\omega_{2, Y M}^{1}$ and $\omega_{2, L}^{1}$ are related to $\operatorname{tr} F_{1}^{2}+\operatorname{tr} F_{2}^{2}$ and $\operatorname{tr} R^{2}$ via descent. Next, using (6.17) and (6.12), the Green Schwarz term (6.3) gives in the small radius limit

$$
\begin{aligned}
S_{G S} & \rightarrow \frac{1}{(4 \pi)^{2}} \int_{M_{10}}\left(\mathrm{~d} B-\omega_{3, Y M}+\omega_{3, L}\right) \wedge X_{7} \\
& =-\frac{1}{(4 \pi)^{2}} \int_{M_{10}} B \wedge X_{8}-\frac{1}{(4 \pi)^{2}} \int_{M_{10}}\left(\omega_{3, Y M}-\omega_{3, L}\right) \wedge X_{7} .
\end{aligned}
$$

The second term is an irrelevant local counterterm: its gauge and local Lorentz variation corresponds to a vanishing $I_{12}$. Such terms can always be added and subtracted. The modified Chern-Simons term (6.19) gives (using (6.17) with $b=1,(6.12),(6.26)$ and integrating by parts on $M_{10}$ )

$$
\widetilde{S}_{C S} \rightarrow-\frac{\beta}{\epsilon^{3}} \sum_{A, B} \int_{M_{10}}\left(\frac{\pi}{(4 \pi)^{2}} B \wedge I_{4, A} \wedge I_{4, B}-\frac{2 \pi}{3} \omega_{3, A} \wedge I_{4, B} \wedge \sum_{C} \omega_{3, C}\right) \int_{S^{1}} \epsilon_{A} \epsilon_{B} \frac{\mathrm{d} x^{10}}{2 \pi r_{0}}
$$

Using again $\beta=\epsilon^{3}$ and the relation

$$
\int_{S^{1}} \epsilon_{A} \epsilon_{B} \frac{\mathrm{d} x^{10}}{2 \pi r_{0}}=\frac{1}{2}\left(\delta_{A B}-\frac{1}{3}\right)
$$

we get

$$
\begin{aligned}
\widetilde{S}_{C S} \rightarrow & -\frac{1}{(4 \pi)^{2}} \int_{M_{10}} B \wedge \frac{\pi}{3}\left(I_{4,1}^{2}+I_{4,2}^{2}-I_{4,1} I_{4,2}\right) \\
& -\frac{2 \pi}{9} \int_{M_{10}}\left(\omega_{3,1}+\omega_{3,2}\right)\left(\omega_{3,1} I_{4,1}+\omega_{3,2} I_{4,2}-\frac{1}{2} \omega_{3,1} I_{4,2}-\frac{1}{2} \omega_{3,2} I_{4,1}\right) .
\end{aligned}
$$

Again, the second term is an irrelevant counterterm. Summing (6.27) and (6.30) we arrive at (cf. $(6.8))$

$$
S_{G S}+\widetilde{S}_{C S} \rightarrow S_{h e t}=-\frac{1}{(4 \pi)^{2}} \int_{M_{10}} B \wedge \hat{X}_{8}\left(R, F_{1}, F_{2}\right)+\text { local counterterms }
$$

where $\hat{X}_{8}\left(R, F_{1}, F_{2}\right)$ is the standard heterotic 8-form given in (6.9). Equation (6.31) is the correctly normalised heterotic anomaly-cancelling term. ${ }^{12}$

\section{Conclusions and discussion}

We have studied carefully and in quite some detail the 1-loop anomalies and their cancellation through inflow from the Chern-Simons and Green-Schwarz terms for the "classic" examples of 5-branes in M-theory and M-theory on $S^{1} / \mathbf{Z}_{2}$, i.e. the strongly coupled heterotic string.

\footnotetext{
${ }^{12}$ In order to facilitate comparison with [10] we note that $\hat{X}_{8}=\frac{1}{(2 \pi)^{3} 4 !} X_{8}^{G S W}$, and $S_{\text {het }}$ as given in (6.31) exactly equals minus the expression given in [10]. The missing minus sign in [10] is due to a sign error related to the subtle issues of orientation, and is corrected e.g. when using the anomaly polynomials as given in [14].
} 
To determine the exact coefficients and signs of the one-loop anomalies, we reviewed the Euclidean results of [2] and discussed the subtleties related to the continuation of their results to Minkowski space. This involved issues of orientation and how the Euclidean and Minkowskian chiralities are related. In the conventions we have chosen, which are the standard Minkowskian string conventions of $[10,11]$ in $D=10$, it turned out that the Minkowski chirality equals minus the Euclidean chirality of [2]. As a result, we found that the anomalous variation of the Minkowskian effective action, due to a field with negative Minkowski chirality, is

$$
\left.\delta \Gamma_{\mathrm{M}}\right|_{1-\text { loop }}=+\int \hat{I}_{2 n}^{1}
$$

where $\hat{I}_{2 n}^{1}$ is related via descent to the $\hat{I}_{2 n+2}$ for the corresponding positive Euclidean chirality field as given in eqs. (4.21)-(4.23). Furthermore, for the 5-brane, we included an ab initio calculation of the chirality of its zero-modes, independent of the conventions and coefficients appearing in the 11-dimensional supergravity action.

Starting from such a general action with arbitrary coefficients for the Chern-Simons and Green-Schwarz terms, cancellation of both, the tangent and normal bundle anomalies of the 5-brane fixes both signs and coefficients up to a possible rescaling of the fields. (Of course, the Chern-Simons term was already fixed from supersymmetry, but it is nice to see that anomaly cancellation requires the same coefficient.) This culminated in our relation $\epsilon^{3}=+\beta$. In particular, the Green-Schwarz and Chern-Simon terms must have the same sign.

When looking at anomaly cancellation for the strongly coupled heterotic string, i.e. for M-theory on $S^{1} / \mathbf{Z}_{2}$ in the "upstairs" approach, we met a surprise. Insisting on defining all quantities properly on $S^{1}$ induces some subtleties considered in [7], but there, a discrepancy by a factor 12 was unnoticed. A factor of 4 is easily accounted for by relating various coefficients between the "upstairs" and "downstairs" approaches. The missing factor 3, however, showed that we must use inflow from a Chern-Simons term, modified by additional contributions induced by the non-trivial Bianchi identity. When all this is taken into account, we obtain cancellation of all anomalies provided the same relation $\epsilon^{3}=+\beta$ between the coefficients of the Green-Schwarz and (modified) Chern-Simons terms holds. Moreover, anomaly cancellation occurs locally on each ten-plane.

As a final check we computed the 10-dimensional anomaly cancelling term of the heterotic string. It receives contributions from both the Chern-Simons and the Green-Schwarz term. The standard $\hat{X}_{8}$ of the heterotic string [10] can only be obtained if the coefficients satisfy $\epsilon^{3}=\beta$. 
In Table 3, we summarise the different constraints between the coefficients and where they come from.

\begin{tabular}{|c||c|}
\hline \multicolumn{1}{|c||}{ origin } & relation \\
\hline \hline supersymmetry & $|\beta|=\alpha^{3 / 2}$ \\
\hline 5-brane tangent bundle anomaly & $\epsilon=\alpha^{1 / 2} \operatorname{sgn}(\beta) \Rightarrow \epsilon^{3}=\alpha^{3 / 2} \operatorname{sgn}(\beta)$ \\
\hline 5-brane normal bundle anomaly & $|\beta|=\alpha^{3 / 2}$ and $\epsilon^{3}=\alpha^{3 / 2} \operatorname{sgn}(\beta) \Rightarrow \epsilon^{3}=\beta$ \\
\hline$S^{1} / \mathbf{Z}_{2}$ & $\epsilon^{3}=\beta$ \\
\hline
\end{tabular}

Table 3: Shown are the different constraints on the coefficients and where they come from.

Furthermore, we want to point out that the gauge and mixed anomalies that arise from compactification of M-theory on singular $G_{2}$-manifolds have a fixed relative sign. These anomalies can only be cancelled through inflow from the Chern-Simons and Green-Schwarz terms if both these terms have the same sign [8].

Taking these constraints into account, a single free parameter remains, related to the possibility of rescaling the $C$-field. Hence, up to such rescalings, all coefficients are fixed as $\alpha=\beta=\epsilon=1$, and the low-energy effective action of M-theory in Minkowski space is given in terms of the 11-dimensional supergravity action $S_{\mathrm{CJS}}$ and the Green-Schwarz term $S_{\mathrm{GS}}$ as

$$
\begin{aligned}
S_{\mathrm{M}-\text { theory }} & =S_{\mathrm{CJS}}+S_{\mathrm{GS}}+\ldots \\
S_{\mathrm{CJS}} & =\frac{1}{2 \kappa^{2}}\left(\int \mathrm{d}^{11} x \sqrt{|g|} \mathcal{R}-\frac{1}{2} \int G \wedge^{*} G-\frac{1}{6} \int C \wedge G \wedge G\right) \\
S_{\mathrm{GS}} & =-\frac{T_{2}}{2 \pi} \int C \wedge X_{8}=-\frac{T_{2}}{2 \pi} \int G \wedge X_{7}=-\frac{1}{\left(4 \pi \kappa^{2}\right)^{1 / 3}} \int G \wedge X_{7},
\end{aligned}
$$

where $+\ldots$ indicates further terms of order higher than $\frac{1}{\kappa^{2}}$, such as those arising from the modifications of the Chern-Simons term discussed in sections 5 and 6 .

\section{Acknowledgements}

Steffen Metzger gratefully acknowledges support by the Gottlieb Daimler- und Karl BenzStiftung. We would like to thank Ruben Minasian for helpful discussions. 


\section{References}

[1] E. Cremmer, B. Julia and J. Scherk, Supergravity theory in 11 dimensions, Phys. Lett. 76B (1978) 409.

[2] L. Alvarez-Gaumé and P. Ginsparg, The structure of gauge and gravitational anomalies, Ann. Phys. 161 (1985) 423, erratum-ibid 171 (1986) 233.

[3] M.J. Duff, J.T. Liu and R. Minasian, Eleven dimensional origin of string/string duality: a one loop test, Nucl. Phys. B452 (1995) 261, hep-th/9506126.

[4] E. Witten, Five-brane effective action in M-theory, J. Geom. Phys. 22 (1997) 103, hep-th/9610234.

[5] D. Freed, J. A. Harvey, R. Minasian and G. Moore, Gravitational anomaly cancellation for M-theory fivebranes, Adv. Theor. Math. Phys. 2 (1998) 601, hep-th/9803205.

[6] P. Hořava and E. Witten, Heterotic and type I string dynamics from eleven dimensions, Nucl. Phys. B460 (1996) 506, hep-th/9510209; P. Hořava and E. Witten, Elevendimensional supergravity on a manifold with boundary, Nucl. Phys. B475 (1996) 94, hep-th/9603142.

[7] A. Bilal, J.-P. Derendinger and R. Sauser, M-theory on $S^{1} / \mathbf{Z}_{2}$ : new facts from a careful analysis, Nucl. Phys. B576 (2000) 374, hep-th/9912150.

[8] A. Bilal and S. Metzger, Anomalies in M-theory on singular $G_{2}$-manifolds, to appear in Nucl. Phys. B, hep-th/0303243.

[9] C. Vafa and E. Witten, A one-loop test of string duality, Nucl. Phys. B447 (1995) 261, hep-th/9505053.

[10] M. B. Green, J. H. Schwarz and E. Witten, Superstring theory, Cambridge University Press, Cambridge, 1987, vol. 2.

[11] J. Polchinski, String Theory, Cambridge University Press, Cambridge, 1998, vol. 2.

[12] B. De Wit, Supergravity, in: Les Houches Summer School "Unity of Fundamental Physics: Gravity, Gauge Theory and Strings", Bachas et al editors, EDP Science 2003, hep-th/0212245. 
[13] D.M. Kaplan and J. Michelson, Zero modes for the $d=11$ membrane and five-brane, Phys. Rev. D53 (1996) 3474, hep-th/9510053.

[14] M. Faux, D. Lüst and B.A. Ovrut, Intersecting orbifold planes and local anomaly cancellation in M-theory, Nucl. Phys. B554 (1999) 437, hep-th/9903028.

[15] J.P. Gauntlett, Branes, calibrations and supergravity, hep-th/0305074.

[16] L. Alvarez-Gaumé and E. Witten, Gravitational Anomalies, Nucl. Phys. B234 (1984) 269.

[17] K.S. Stelle, BPS branes in supergravity, in High energy physics and cosmology, Trieste 1997, 29, hep-th/9803116.

[18] S.P. de Alwis, A note on brane tension and M-theory, Phys. Lett. B388 (1996) 291, hep-th/9607011.

[19] R. Bott and A.S. Cattaneo, Integral invariants of 3-manifolds, J. Diff. Geom. 48 (1998) $91, \mathrm{dg}-\mathrm{ga} / 9710001$. 